\title{
Dynamic Argumentation in UbiGDSS
}

\author{
João Carneiro • Diogo Martinho • \\ Goreti Marreiros • Amparo Jimenez . \\ Paulo Novais
}

Received: date / Accepted: date

\begin{abstract}
Supporting and representing the group decision-making process is a complex task that requires very specific aspects. The current existing argumentation models cannot make good use of all the advantages inherent to group decision-making. There is no monitoring of the process or the possibility to provide dynamism to it. These issues can compromise the success of Group Decision Support Systems if those systems are not able to provide freedom and all necessary mechanisms to the decision-maker. We investigate the use of argumentation in a completely new perspective that will allow for a mutual understanding between agents and decision-makers. Besides this, our proposal allows to define an agent not only according to the preferences of the decisionmaker but also according to his interests towards the decision-making process. We show that our definition respects the requirements that are essential for groups to interact without limitations and that can take advantage of those interactions to create valuable knowledge to support more and better.
\end{abstract}

Keywords Argumentation · Automatic Negotiation · Multi-Agent Systems · Group Decision Support Systems · Ubiquitous Computing

João Carneiro · Diogo Martinho · Goreti Marreiros

GECAD - Research Group on Intelligent Engineering and Computing for Advanced Innovation and Development, Institute of Engineering, Polytechnic of Porto, Porto 4200-072, Portugal

E-mail: jomrc@isep.ipp.pt

João Carneiro · Paulo Novais

ALGORITMI Centre, University of Minho, Guimarães 4800-058, Portugal

Amparo Jimenez

Universidad Pontificia de Salamanca 


\section{Introduction}

There are several reasons which lead decisions to be made in group: to improve the quality of the decision, to share workloads, to gain support among stakeholders, to train less experienced group members and due to the majority of organograms existing nowadays (Bell, 1985; Huber, 1984). It has been proven that groups obtain performances that are qualitatively and quantitatively superior to the individual performance (Hill, 1982; Shaw, 1932). However, in order to take advantage of the benefits behind group decision-making it is necessary to create conditions in which groups can perform certain tasks, such as generating ideas and solutions through group interaction (Watson et al, 1991; Hackman and Morris, 1974). It is considered that with the group decisionmaking process, members will enhance the ability to learn and stimulate the cognition level (Lamm and Trommsdorff, 1973; Osborn, 1963). Moreover, studies (Hill, 1982) show that cognitive stimulation helps people to think of new ideas, "unique combination or sub ideas, or a complex solution whose total value is greater than the sum of its parts".

Group Decision Support Systems (GDSS) appeared with the goal to support groups in the decision-making process (DeSanctis and Gallupe, 1987). They have been studied over the past 30 years, and have become one of the most important investigation topics in the area of Artificial Intelligence $(\mathrm{Hu}-$ ber, 1984; DeSanctis and Gallupe, 1987, 1985; Marreiros et al, 2010). With the appearance of global markets, the growth of multinational enterprises and a more global vision of the planet, we easily find chief executive officers and top managers (decision-makers) spread around the world, in countries with different time zones (Grudin, 2002). Nowadays, the study of GDSS has been oriented to support groups with members that cannot gather at the same place and at the same time (Kwon et al, 2005). In order to provide an answer and operate correctly in this type of scenarios the traditional GDSS have evolved to what we identify today as Ubiquitous Group Decision Support Systems (UbiGDSS). The UbiGDSS support the decision-making process by using the main characteristics of ubiquity ("anytime and "anywhere") (Kwon et al, 2005; Daume and Robertson, 2000). For UbiGDSS to be advantageous they must share a number of features that put them above all the traditional GDSS. In order to support the decision-making process in an ubiquitous context it makes sense that UbiGDSS should: allow automatic negotiation, represent interests of decision-makers, allow the existence of a process, generate ideas, discuss points of view, etc (Carneiro et al, 2015d, 2014). However, something is wrong with GDSS that we know today. Everyone has acknowledged the benefits of this type of systems; however, we do not actually see these systems being used in reality and it is not because the concept is still fresh. In fact, it is impossible to identify the reasons that lead to their absence and why they are not accepted in the industry sector nowadays. On the other hand, what we know is that most of artificial intelligence techniques proposed in the literature that could be used in UbiGDSS go against what are considered to be the benefits of group decision-making. 
In this work, we propose a refreshing look into the concept of what and how should be the artificial intelligence mechanisms that compose an UbiGDSS. For that, we introduce a dynamic argumentation framework, which provides the system with the features that are necessary for decision-makers to take advantage of the benefits of group decision-making. Our proposal intends to follow decision-makers throughout the entire process. Our approach allows a group of decision-makers, where each agent represents a decision-maker, to seek a possible solution to a problem (choosing between several alternatives) while taking into account all the preferences of the decision-makers. Besides this, and considering that the decision-makers can understand the conversation performed between agents, those agents will also be able understand the new arguments created and exchanged between decision-makers. These new arguments can be processed and used by agents not only to advice decision-makers, but also to find solutions throughout the decision-making process.

With this work, we intend to change the current perspective on UbiGDSS. The idea is that decision-makers see the system as something that is easy to use and that guides them throughout the decision-making process, helps them to achieve better decisions, is clear, does not inhibit the generation of new ideas, allows decision-makers to create new arguments, etc. Our approach intends, above everything else, to promote group interaction and help achieving the typical high quality decisions that are common in face-to-face meetings. We believe that our proposal will allow to overcome many issues while supporting ubiquitous group decision-making, but mainly (1) make it possible for both agents and decision-makers to use a dialogue which is clear to everyone involved in the decision-making process (2) let both agents and decision-makers to take advantage of the knowledge which is generated and (3) not force decision-makers with information formulation patterns which may compromise the usability of the entire system as well as the process.

The rest of the paper is organized in the following order: Section 2 exposes the literature review of most recent works related to argumentation-based negotiation. In Section 3, our approach is presented and the definition of the argumentation model is described. In Section 4, we can find the evaluation and results of this work and in Section 5 the discussion is presented. Finally, some conclusions are taken in Section 6, along with the work to be done hereafter.

\section{Related Work}

In the literature there are various negotiation models adapted to decisionmaking (Gordon and Karacapilidis, 1997; Ito and Shintani, 1997; Karacapilidis and Papadias, 2001; Kudenko et al, 2003; Marreiros et al, 2010). To study argumentation (in the perspective of automated negotiation) it is crucial to read Dung (1995) "On the acceptability of arguments and its fundamental role in non monotonic reasoning, logic programming and n-person games", Kraus et al (1998) "Reaching agreements through argumentation: a logical model and implementation", Sierra et al (1997) "A framework for argumentation-based 
negotiation", and other works in this area. However, in this document, only the most recent literature will be reviewed.

Heras et al (2010) analysed how Argumentation Schemes Theory can be used to formalise and structure on-line discussions and user opinions. They considered a Social network to be an abstraction of social structures that connects individuals or organizations. They distinguished implicit and explicit social networks and presented several features that could be used to define those networks (such as purpose, nodes, roles, etc.). They also identified three main advantages of applying argumentation schemes to social network business (to provide a formal structure to opinions and recommendations, to provide a way of evaluating user opinions and recommendations, and to provide a formal structure to the dialogue). As a final remark, they believe that argumentation can enhance business driven social networking.

Sánchez-Anguix et al (2011) proposed and studied the performance of several intra-team strategies that could be used by negotiation teams. These strategies define how the communication is carried out between team members and how decisions can be made (which requests are sent to the opponent and what mechanisms are used for the opponent to accept or refuse the request). They concluded that the negotiation environment (diversity, negotiation on time, concession strategy) affects greatly the performance of the intra-strategy that is chosen. They also referred to the fact of their approach being new in the literature and that they could further improve its performance by considering more environmental conditions (opponent strategies, non-static team memberships, etc.)

Van der Weide et al (2011) published a scientific work where they proposed a formalism to argue on a meta-level about which argument an agent should select in a given persuasion dialogue. They claimed their approach is easier to design compared to a purely quantitative approach, it allows the use of criteria that are partial and/or incommensurable and it is possible for the agent to explain why a certain argument is selected.

El-Sisi and Mousa (2012) presented a paper where they analysed the benefits of argumentation-based negotiation (ABN) against proposal-based approaches (PBA). They considered the possibility to exchange additional information as the biggest advantage of ABN. They concluded that the argumentation increased the quality of the agreements. Another very important fact was that all the failed examples in PBA become accepted in ABN. They stated the $\mathrm{ABN}$ is much better than PBA in terms of the quality of the agreements and the quantity of unsuccessful negotiations.

Booth et al (2012) addressed a work where they proposed a way to measure and quantify the disagreement in argument-based reasoning. They highlighted the need for an approach to measure the distance between different positions. To achieve this objective, they presented several different distance functions. As future work, they pretend to continue studying this issue and apply it to the problems of revision and judgement aggregation in argumentation.

Müller and Hunter (2012) published a work proposing the use of argumentation to satisfy the needs to support the decision-maker and to document 
the reasons behind decisions. Their framework consists in 2 different steps, the first step is related to the creation of arguments and the second step is to consider arguments created and choose the best alternative. Although their work has a strong relation with industry they did not present any validation.

Bonzon et al (2012), presented an extended abstract where they proposed a reasoning mechanism "that allows negotiating agents to take into account information about their counterparts". To validate their approach, they ran a wide set of experiments, where they compared the negotiation strategy with and without their approach. They concluded that the new approach improves the performance of the system (namely the length of the negotiation and the quality of the agreement).

Wyner et al (2012), presented a notable work about abstract argumentation and argumentation schemes. Their idea was to integrate the argumentation schemes with abstract argumentation through a functional language. They did not run any tests until then, however they intend to "implement argumentation schemes in a database for web-based applications and Functional or Logic Programs that instantiate the argumentation schemes, as well as to generate arguments, calculate attacks, and determine extensions".

Heras et al (2013) proposed an argumentation framework for Multi-Agent Systems using case-based reasoning. In their framework, the agents use arguments to reach an agreement. During the dialogue the arguments can be classified as acceptable, unacceptable, or undecided. They evaluated their system in terms of knowledge which agents have about the social context of their partners, performance, percentage of agreement, and influence on the amount of argumentation knowledge of each agent. They concluded that arguments used by experts are usually preferred; agents using their argumentation framework usually provide more accurate solutions and achieve higher percentages of agreement compared to agents who do not use it; and the more knowledge an agent possess about the context of the argumentation process, the number of agreements as well as the number of agreeing agents will also increase. However, they also pointed to the fact that achieving a consensus could be difficult in case all agents had the same amount of knowledge and therefore had the same persuasive power.

Fan et al (2013) published a scientific work about assumption-based argumentation for decision-making including preferences over goals. One of the most interesting points of this work is the possibility to define preferences over combined goals. They applied their approach to a case study in medical research area and they obtained satisfactory results. They claimed that their approach is easy to apply to other domains.

Parsons et al (2013) produced a research where they included the concept of trust in their argumentation-based approach. The focus of their work is to support the decision-making in scenarios where trust is variable. In their work, they used an example of military decision-making where they applied their argumentation-based approach. However, no results were presented, nor any kind of evaluation. 
Fan and Toni (2013) presented a relevant work about assumption-based argumentation. In this work they presented two different frameworks to represent decision-making. They introduced very interesting notions of dominant decisions: strongly dominant, dominant and weakly dominant. In one of their frameworks they included the possibility to define preferences over goals. The main advantage of their work is the possibility to explain the selected decisions through argumentation-based justification. However, they considered important to continue the studies for decision-making with preference.

Fan et al (2014) addressed a work about assumption-based argumentation. They considered that most works found in the literature related to argumentation-based decision-making did not pay attention to decision-making amongst multiple agents. The main contribute of this paper is how it covers the entire decision-making process: decision frameworks, argumentation-based computation, dialogues and a real world application with implementation. They concluded that successful dialogues generate good decisions.

Marey et al (2014a) published a paper where they included uncertainty in argumentation-based negotiation. This work came in the sequence of the one presented in Marey et al (2014b). They conducted a case study (buyer/seller scenario) based on their proposed approach and concluded that with their techniques negotiating agents achieve better results than non-negotiating agents.

Marey et al (2015) addressed the problematic of negotiation in ambiguous conditions. They proposed a framework capable of tackling the agents' uncertainty. Their framework allows to measure agents' uncertainty and helps them to select better choices among the available possibilities. They ran a case study (Buyer/Seller) and they considered their results were better than others that use pure argumentation without considering uncertainty.

Sklar et al (2016) conducted a study in order to evaluate the effectiveness of "ArgTrust". The "ArgTrust" is an interactive application developed to help human users in the decision-making process (Parsons et al, 2013). In this work, they tested the "ArgTrust" in an ambiguous and complex scenario, where participants completed pre-, mid- and post-surveys based in their understanding about a certain scenario, before and after use "ArgTrust". The major conclusion is that "ArgTrust" has contributed to help users "consider their decisions more carefully".

\section{The Argumentation Model}

In this paper, we consider the following structure of a decision problem: there are a set of possible alternatives $A$, a set of criteria $C$, and a set of agents $A g$, such that an alternative $a \in A$ has a value for all the defined criteria $C$. The decision problem has a defined communication language $\mathcal{L}_{c}$ which allows agents $A_{g}$ to communicate. In order to operate with the defined $\mathcal{L}_{c}$, there is a set of algorithms $\mathcal{L}_{a}$, which specify for each locution $\varphi \in \mathcal{L}_{c}$ its effect. The 
relations between alternatives, criteria, agents, communication language and algorithms jointly form a decision system, represented as follows:

Definition 1 A decision system $\left(C, A, A_{g}, \mathcal{L}_{c}, \mathcal{L}_{a}\right)$, consists of:

- a set of criteria $C=\left\{c_{1}, c_{2}, \ldots, c_{n}\right\}, n>0$;

- a set of alternatives $A=\left\{a_{1}, a_{2}, \ldots, a_{m}\right\}, m>0$;

- a set of agents $A_{g}=\left\{a g_{1}, a g_{2}, \ldots, a g_{k}\right\}, k>0$;

- a communication language $\mathcal{L}_{c}$, consisting of a set of all locutions;

- a set of algorithms working as regulation $\mathcal{L}_{a}$ for $\mathcal{L}_{c}$, specifying for each locution $\varphi \in \mathcal{L}_{c}$ its effect.

Rule 1 Each alternative is related with each criterion. There cannot be an existing alternative with values for criteria that is not considered in the problem.

Definition 2 A criterion $c_{i}=\left\{i d_{c_{i}}, v_{c_{i}}, m_{c_{i}}\right\}$ consists of:

$-\forall c_{i} \in C, i \in\{1,2, \ldots, n\}$;

- $i d_{c_{i}}$ is the identification of a particular criterion;

$-v_{c_{i}}$ is the value of a particular criterion (Numeric, Boolean or Classificatory);

- $m_{c_{i}}$ is the greatness associated with the criterion (Maximization, Minimization, Positivity, Negativity and Without Value).

Example 1 For the previous example, let us consider three criteria: Price, Transmission, Air Conditioning. Each criterion is defined as follows:

- $c_{1}=\{$ Price, Numeric, Minimization $\}$

$-c_{2}=\{$ Transmission, Classificatory, WithoutValue $\}$

$-c_{3}=\{$ AirConditioning, Boolean, Positivity $\}$.

Definition 3 An alternative $a_{i}=\left\{i d_{a_{i}},\left[c_{1_{a_{i}}}, c_{2_{a_{i}}}, c_{n_{a_{i}}}\right]\right\}$ consists of:

- $\forall a_{i} \in A, i \in\{1,2, \ldots, n\}$;

- $i d_{a_{i}}$ is the identification of a particular alternative;

$-\left[c_{1_{a_{i}}}, c_{2_{a_{i}}}, \ldots, c_{n_{a_{i}}}\right]$ is the instantiation of each criterion.

Example 2 For the previous example, let us consider three alternatives. Each alternative is defined as follows:

$-a_{1}=\{$ car $1,[10000$, automatic, no $]\}$;

$-a_{2}=\{$ car $2,[15000$, manual, yes $]\}$

$-a_{3}=\{$ car $3,[12500$, manual, no $]\}$.

The way each criterion is defined, allows an agent to know (in the previous example) that $c_{1_{a_{1}}}>c_{1_{a_{2}}} \wedge c_{1_{a_{1}}}>c_{1_{a_{3}}} \wedge c_{1_{a_{2}}}>c_{1_{a_{3}}}$, and $c_{2_{a_{1}}} \neq c_{2_{a_{2}}} \wedge c_{2_{a_{1}}} \neq$ $c_{a_{a_{3}}} \wedge c_{2_{a_{2}}}=c_{1_{a_{3}}}$ and $c_{3_{a_{2}}}>c_{3_{a_{1}}} \wedge c_{3_{a_{2}}}>c_{3_{a_{3}}} \wedge c_{3_{a_{1}}}=c_{3_{a_{3}}}$. 
An agent has a special structure that allows him to act according to the interests of the decision-maker he represents. Besides the agent's identification code, the decision-maker can also define the agent's style of behaviour for a certain time interval. The decision-maker may change that style of behaviour whenever he thinks to be appropriate. The proposed styles of behaviour (previously defined in (Martinho et al, 2015; Carneiro et al, 2015a)) allow the agent to act according to 4 dimensions: activity, resistance to change, concern for other and concern for self. Agents also include a protocol where it is defined a set of available locutions available to $a g_{i}$ in a time instant of $t$. An agent also holds the information about the evaluation done by the decision-maker about the preference of each alternative and the importance given to each considered criterion. In order to perform these configurations, the decision-maker can use a template that was proposed in (Carneiro et al, 2015c,b). This template is very easy and fast to configure and can be used to obtain all this information with mechanisms that make it easier for the decision-maker to express his opinion and evaluations. The agent also includes a list of objectives to pursue after. This list is ordered (preference relation $\geq$ on the set $O_{a g_{i}}$ ) and may contain alternatives and criteria. An agent can have as the main objective to achieve alternative $a_{1}$ as the final decision or to achieve an alternative with the best $c_{1}$ as possible, which in our example would correspond to the "the less expensive car". Agents may keep adding new objectives to their list of objectives throughout the process as well as reordering existing ones.

Example 3 An agent $a g_{i}$ with the defined behaviour 'Obliging' can start a discussion with the main objective to achieve the alternative, which the decisionmaker (that he represents) chose as the most preferred (in the problem configuration). However, if more and more agents support another alternative, and since that agent has a high level of "concern for others" he may reorder his objectives and choose other alternatives as his main objective.

Definition 4 An agent $a g_{i}=\left\{i d_{a g_{i}}, u i d_{a g_{i}}, \beta_{a g_{i}}, P r_{a g_{i}}, C_{a g_{i}}, A_{a g_{i}}, O_{a g_{i}}, K_{a g_{i}}\right\}$ consists of:

$-\forall a g_{i} \in A g, i \in\{1,2 \ldots, n\}$;

- $i d_{a g_{i}}$ is the identification of a particular agent;

- $u i d_{a g_{i}}$ is the identification of the decision-maker represented by the agent $a g_{i}$

- $\beta_{a g_{i}}$ is the agent's behaviour (Dominating, Compromising, Obliging, Integrating, Avoiding and No Style);

- $\operatorname{Pr}_{a g_{i}}$ is the agent's protocol for $\mathcal{L}_{c}$, specifying the 'legal' moves at each instant. A protocol on $\mathcal{L}_{c}$ is a set of locution available to $a g_{i}$, where $\operatorname{Pr}_{a g_{i}} \subseteq$ $\mathcal{L}_{c}$;

- $C_{a g_{i}}$ is the agent's evaluation of each criterion, $C_{a g_{i}}=\left\{E_{c_{1}}, E_{c_{2}}, \ldots, E_{c_{n}}\right\}, E v_{c_{i}} \in$ $\{[0,1], \perp\}$;

- $A_{a g_{i}}$ is the agent's evaluation of each alternative, $A_{a g_{i}}=\left\{E_{a_{1}}, E_{a_{2}}, \ldots, E_{a_{n}}\right\}, E v_{a_{i}} \in$ $\{[0,1], \perp\}$

- $O_{a g_{i}}$ is the set of agent's objectives, $O_{a g_{i}} \subseteq A \cup C$, preference relation $\geq$ on the set $O_{a g_{i}}$; 
- $K_{a g_{i}}$ is the agent's knowledge, where he can access the list of all sent and received messages, as well as the preferences of other agents, according to the knowledge he possess in a certain time instant of $t$.

$O_{a g_{i}}$ can be reordered using the following formula:

$$
A_{\text {Result }_{o_{i}}}=\frac{o_{i} * C S+\left(\frac{N S}{N D}\right) \times C O}{C S+C O}
$$

Where:

- $o_{i}$ is the assessment done to the objective $i$ for which the result is being measured;

- $C S$ is the value of Concern for Self $[1,2,3]$;

- NS is the current number of agents supporting $A l t_{x}$;

- $N D$ is the total number of participating agents;

- $C O$ is the value of Concern for Others $[1,2,3]$.

Formula 1 reflects the importance of the objective to an agent with a certain style of behaviour, while he takes into account the probability to achieve that same objective. Our proposal intends to (besides helping to achieve a consensus) help the group to take advantage of the benefits related to group decision-making and be able to achieve decisions with more quality. Therefore, an important metric is to relate the level of consensus with the final level of satisfaction that is achieved by each decision-maker.

Definition 5 A behaviour $\beta_{i}=\left\{R c_{\beta_{i}}, A l_{\beta_{i}}, C s_{\beta_{i}}, C o_{\beta_{i}}\right.$ consists of (according to (Martinho et al, 2015)):

- $R c_{\beta_{i}}$ is the agent's resistance to change dimension value;

- $A l_{\beta_{i}}$ is agent's activity level dimension value;

- $C s_{\beta_{i}}$ is the agent's Concern for Self dimension value;

- $C o_{\beta_{i}}$ is the agent's Concern for Others dimension value.

Definition 6 A locution $\varphi_{i}=\left\{i d_{\varphi_{i}}, T p_{\varphi_{i}}, T x_{\varphi_{i}}, C t_{\varphi_{i}}, V r_{\varphi_{i}}, D r_{\varphi_{i}}, D m_{\varphi_{i}}, A v_{\varphi_{i}}\right\}$ consists of:

$-i \in\{1,2, \ldots, n\}$

$-i d_{\varphi_{i}}$ is the locution's id (unique);

$-T p_{\varphi_{i}}$ is the locution's type (Question, Statement and Request);

$-T x_{\varphi_{i}}$ is the text associated to the locution;

- $C t_{\varphi_{i}}$ is the locution's context (Alternative, Criterion or Without Context);

$-V r_{\varphi_{i}}$ is the set of variables associated to the locution (Alternative or Criterion);

- $D r_{\varphi_{i}}$ is the direction associated to the locution (infavor, against, null);

- $D m_{\varphi_{i}}$ is the locution's domain (General or Specific);

- $A v_{\varphi_{i}}$ is the locution's state (Available or Not Available).

Rule 2 Whenever a locution is added to $P r_{a g_{i}}$ in the time instant $t$ its state will be $A v_{\varphi_{i}}^{t}, A v=$ Available. 
Rule 3 Whenever a locution is used at a time instant $t$ its state will change to $A v_{\varphi_{i}}^{t}, A v=$ Not Available.

Rule 4 Whenever a locution $D m_{\varphi_{i}}, D m=$ Specific is added to $P r_{a g_{k}}$ at the time instant $t$, then $\forall D m_{\varphi_{i}} \in \mathcal{L}_{D m} \subset P r_{a g_{k}}, D m=$ General and its state will be $A v_{\varphi_{i}}^{t}, A v=$ Available.

Rule 5 For any locution $\varphi_{j} \in \mathcal{L}_{s p_{c_{i}}} \wedge c_{i} \subset V r_{\varphi_{j}}$ there cannot be another locution $\varphi_{k}$ where $c_{i} \subset V r_{\varphi_{k}} \wedge \varphi_{k} \notin \mathcal{L}_{s p_{c_{i}}}$.

Rule 6 For any locution $\varphi_{j} \in \mathcal{L}_{s p_{a_{i}}} \wedge a_{i} \subset V r_{\varphi_{j}}$ there cannot be another locution $\varphi_{k}$ where $a_{i} \subset V r_{\varphi_{k}} \wedge \varphi_{k} \notin \mathcal{L}_{s p_{a_{i}}}$.

Some examples of possible locutions are specified in Table 1 (in our experiments we are dealing with 18 different locutions).

Table 1: Considered Locutions (some examples)

\begin{tabular}{|c|c|c|c|c|c|}
\hline Locution & Type & Text & Context & Variables & Domain \\
\hline $\begin{array}{l}\text { Criteria } \\
\text { General } \\
\text { Preference }\end{array}$ & Statement & $\begin{array}{l}\text { "For me the most } \\
\text { important criteri- } \\
\text { on/a is/are } 1,2, \\
\ldots, \text { n" }\end{array}$ & Criterion & $\begin{array}{l}\text { Criterion } 1, \\
2, \ldots, \mathrm{n}\end{array}$ & General \\
\hline $\begin{array}{l}\text { End of Par- } \\
\text { ticipation }\end{array}$ & Statement & $\begin{array}{l}\text { "I have nothing } \\
\text { more to say" }\end{array}$ & $\begin{array}{l}\text { Without } \\
\text { Context }\end{array}$ & - & General \\
\hline $\begin{array}{l}\text { Alternatives } \\
\text { Individual } \\
\text { Preference }\end{array}$ & Question & $\begin{array}{l}\text { "Who prefers the } \\
\text { alternative/s n?" }\end{array}$ & Alternative & $\begin{array}{l}\text { Alternative } \\
1,2, \ldots, \mathrm{n}\end{array}$ & Specific \\
\hline$\ldots$ & $\ldots$ & $\ldots$ & $\ldots$ & $\ldots$ & $\ldots$ \\
\hline
\end{tabular}

Definition 7 A message $\psi_{i}=\left\{i d_{\psi_{i}}, \varphi_{\psi_{i}}, t r_{\psi_{i}}, \alpha_{\psi_{i}}, e n_{s_{\psi_{i}}}, E n_{r_{\psi_{i}}}\right\}$ consists of:

$-i \in\{1,2, \ldots, n\}$;

$-i d_{\psi_{i}}$ is the conversation code;

$-\varphi_{\psi_{i}}$ is the locution sent in the message;

$-t r_{\psi_{i}}$ is the target associated with the message (can be null or be another message);

$-\alpha_{\psi_{i}}$ is the justification associated to the locution (can be an argument or can be null);

- $e n_{s_{\psi_{i}}}$ is the agent/user who sent the message;

- $E n_{r_{\psi_{i}}}$ is the set of agents/users who will receive the message (can be 1 or $*)$.

Rule 7 For any message $\psi$ create by a decision-maker $D r_{\varphi_{\psi}}, D r \neq$ null. This means that the message's locution can only be either infavor or against $V r_{\varphi_{\psi}}$.

Definition 8 An argument $\alpha_{i}=\left\{i d_{\alpha_{i}}, t x_{\alpha_{i}}, V r_{\alpha_{i}}\right\}$ consists of:

$-i \in\{1,2, \ldots, n\}$ 


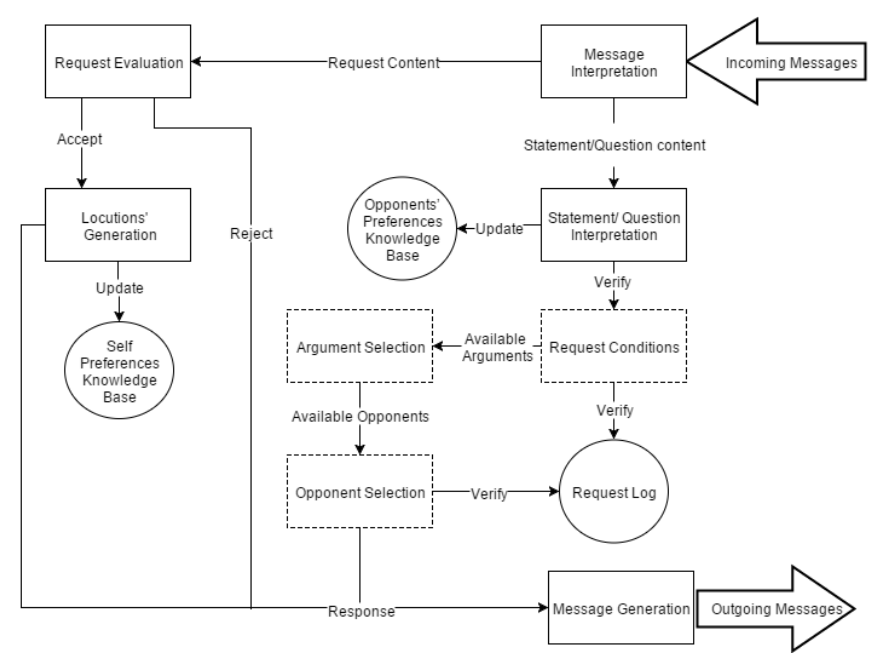

Fig. 1: Agent's Communications Workflow

- $i d_{\alpha_{i}}$ is the identification of a particular argument;

- $t x_{\alpha_{i}}$ is the text associated to a particular argument;

- $V r_{\alpha_{i}}$ is the set of variables associated to a particular argument (can contain alternatives and criteria).

Fig. 1 represents the workflow proposed to use the argumentation model definition that is presented in this work. This workflow represents the communications and the interactions which can be performed by agents. The process is very clear and allows the decision-makers to understand the interactions and communications that happen between agents.

Proposition 1 The system is finite.

Proof The size of the list of objectives of an agent $a g_{i}$, which includes criteria and alternatives will be $\left|O_{a g_{i}}\right| \leq|A| \cup|C|$, that corresponds at best to the entire set of all criteria and alternatives. This means that at a certain time instant $t$ agent $a g_{i}$ may use legally $\mathcal{L}_{C t}^{t}, C t=$ Alternative $\vee$ Criterion locutions. Whenever a new objective is added (for example a new alternative) the $\mathcal{L}_{s p_{a_{j}}}$ is added to $\operatorname{Pr}_{a q_{i}}$. If in a time instant $t+x$ all the criteria and alternatives were added to $P r_{a g_{i}}$ then $P r_{a g_{i}}^{t+x}=\mathcal{L}_{c}$. Since $\forall D m_{\varphi_{j}} \in \mathcal{L}_{D m} \subset P r_{a g_{i}}^{t+x}, D m=$ Specific can only be used once, even if $P r_{a g_{i}}$ size may increase throughout the process, the system will be always finite.

Up until now we have presented the definition for the proposed argumentation model only considering the agents' point of view. From this point forward all the definitions presented will be directed towards to the interactions between decision-makers. However these definitions are an extent of what has been proposed so far which will allow both agents and humans to use the same model definition. 


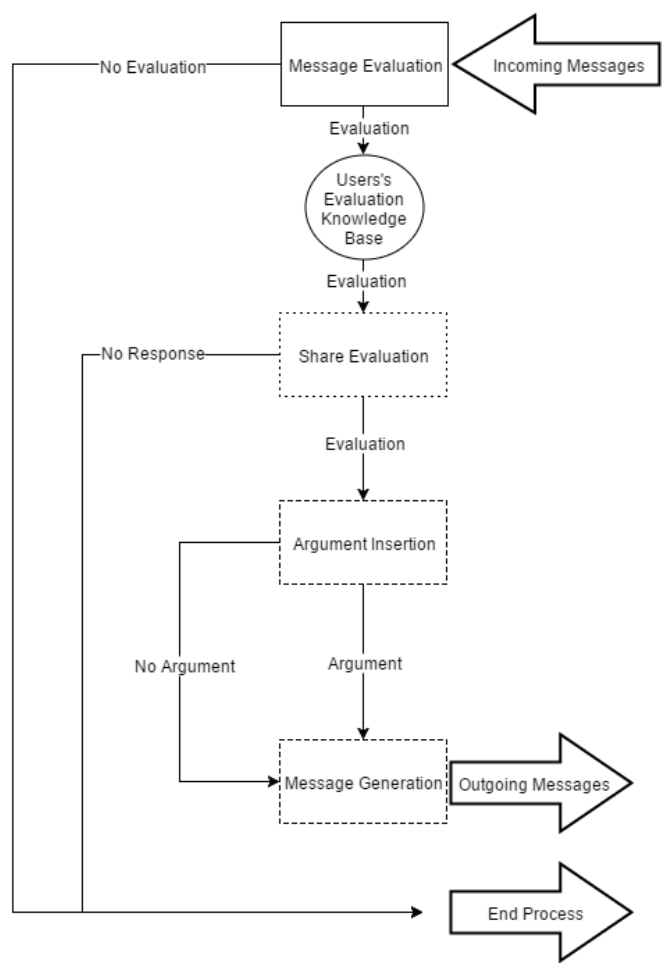

Fig. 2: Decision-Makers' Communications Workflow

Decision-makers, compared to agents, can also create messages that will hold the new knowledge as well as a new set of dynamic arguments. We can imagine an UbiGDSS as something that can be used in all sort of electronic devices and that allows quick interactions. An example of a quick interaction would be the easiness in which we can leave a "Like" in a Facebook photo. Our approach allows decision-makers to create messages that include arguments that are infavor or against. Each created message may lead to $n-1$ messages, where $n$ is the number of decision-makers evolved in the process. Decisionmakers can argue against a message through the use of an attack or argue in favour of a message through the use of a reinforcement. The way the model is defined allows the message content to be of any sort of format like text or voice, since that information is irrelevant to the agent. A decision-maker may evaluate messages sent by other decision-makers, and this will allow agents to understand human interactions and the impact of every conversation. Fig. 2 represents the workflow proposed for all the interactions and communications that happen between decision-makers.

Example 4 (message pro) $D r_{\varphi_{\psi}}, D r=$ infavour. That means something positive related to $V r_{\varphi_{\psi}}$. Such message is said pro the $V r_{\varphi_{\psi}}$. For our previ- 
ous example a message $\psi$ pro could have $V r_{\varphi_{\psi}}, V r=a_{1} \wedge T x_{\alpha_{\psi}}, T x=$ "1st maintenance service is free".

Example 5 (message cons) $D r_{\varphi_{\psi}}, D r=$ against. That means something negative related to $V r_{\varphi_{\psi}}$. Such message is said cons the $V r_{\varphi_{\psi}}$. For our previous example a message $\psi$ cons could have $V r_{\varphi_{\psi}}, V r=c_{3}, c_{3_{a}}=n o \wedge T x_{\alpha_{\psi}}, T x=$ "The high temperatures in our area will damage the product in a car without air conditioning".

Definition 9 Let $\Psi=\left\{\psi_{1}, \psi_{2}, \ldots, \psi_{n}\right\}, n>0$ denote a finite set of $n$ messages that are exchanged during a human discussion.

Let us now define two functions that relate the messages in favour or against an $a_{i} \in A, c_{j} \in C, A_{k} \subseteq A$ or $C_{l} \subseteq C$ (Let us consider $X \asymp a_{i} \cup c_{j} \cup A_{k} \cup C_{l}$ ):

- Finfavour $: X \rightarrow \forall \psi \in \Psi, D r_{\varphi_{\psi}}=$ infavour, is a function that returns the messages in favour of $X$. Such messages are said pro the $X$;

- Fagainst $: X \rightarrow \forall \psi \in \Psi, D r_{\varphi_{\psi}}=$ against, is a function that returns the messages against $X$. Such messages are said cons the $X$.

Rule 8 A message is either in favour or against $X$. It cannot be both, so: $\forall \psi \in \Psi$ s.t. $\neg\left(\psi \in F_{\text {infavour }}(X) \wedge \psi \in F_{\text {against }}(X)\right)$.

It is obvious that we are dealing with a context where we can easily find many conflicts of interests. This means that even if decision-makers have the collective goal to achieve the best possible solution for the group, they will still try to persuade each other to accept their preferred alternatives that they consider to be the best solution for the problem. This leads to different views on how to solve the problem (which is an advantage of group decision-making). In order to represent this situation, our proposal allows decision-makers to evaluate messages created by other decision-makers.

Our messages may have strengths of various weights. But those strengths depend on each decision-maker evaluation. This means that in our proposal a decision-maker can consider an argument to be very strong (or important) while another decision-maker may consider that same argument to be completely irrelevant. That strength allows arguments to rank-order each alternative according to the decision-maker point of view. We believe that this evaluation should be made in a very simple way, similarly to how a person leaves a "Like" in a social network. With the definition here proposed it is possible to filter all the information towards decision-makers' evaluations and the messages interdependency.

Definition 10 A message evaluation $\xi=\left\{e n_{\xi_{i}}, \psi_{\xi_{i}}, e v_{\xi_{i}}\right\}$ consists of:

$-i \in\{1,2, \ldots, n\}$

- $e n_{\xi_{i}}$ is the user who performed the evaluation;

$-\psi_{\xi_{i}}$ is the message being evaluated;

$-e v_{\xi_{i}}$ is the evaluation mode $[-1,1]$. 


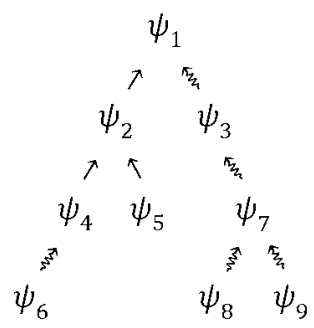

Fig. 3: Representation of $(n-1)$ ary tree messages

Definition 11 Let $\Xi=\left\{\xi_{1}, \xi_{2}, \ldots, \xi_{n}\right\}$ denote a finite set of $n$ evaluations that are made during a human discussion.

Let us now define two functions that return all messages approving or disapproving a message $\psi_{i}$ :

- $F_{\text {approval }}: \psi_{i} \rightarrow \forall \xi \in \Xi, \psi_{\xi}=\psi_{i} \wedge e v_{\xi_{i}}>0$, is a function that returns all messages approving $\psi_{i}$;

- $F_{\text {dissaproval }}: \psi_{i} \rightarrow \forall \xi \in \Xi, \psi_{\xi}=\psi_{i} \wedge e v_{\xi_{i}}>0$, is a function that returns all messages disapproving $\psi_{i}$.

In our proposal, the notion of attack is intrinsically directional: if $\psi_{1}$ attacks $\psi_{2}$ this corresponds to the fact that $\psi_{1}$ has the power to affect $\psi_{2}$, and not vice versa. Likewise, if $\psi_{1}$ reinforce $\psi_{2}$ this corresponds to the fact that $\psi_{1}$ has the power to support $\psi_{2}$. Notice that, we consider the attacks and reinforcements between messages and not between arguments. However, when a decisionmaker decides to perform an attack or a reinforcement, it is mandatory to insert the justification, so a message has mandatorily an argument included. We represent an attack through $\psi_{1} \rightarrow \psi_{2}$ and a reinforcement through $\psi_{1} \rightsquigarrow$ $\psi_{2}$. Each message can result in $n-1$ messages, where $n$ is the number of decision-makers evolved in the process. A group of messages with the same $i d_{\psi_{i}}$ can be represented through a $(n-1)$ ary tree (Fig. 3).

A message $\psi_{i}$ can only affect a message $\psi_{j}$ only if there is a directed path from $\psi_{i}$ to $\psi_{j}$. That means (considering Fig. 3) if a decision-maker evaluates positively the $\psi_{9}$, his evaluation is also affecting $\psi_{9}, \psi_{7}, \psi_{3}$ and $\psi_{1}$. On the other hand (and again considering Fig. 3) if the decision-maker evaluates positively $\psi_{4}$, although $\psi_{4}$ has the same sign as $\psi_{3}$, we cannot correlate the messages.

Let us now define two functions that relate a message $\psi_{1}$ to the messages reinforcing it and to the messages attacking it:

- Freinforcement $: \psi_{1} \rightarrow \forall \psi \in \Psi, t r_{\psi}=\psi_{1} \cap F_{\text {approval }}\left(\psi_{1}\right)$, is a function that returns the messages reinforcing $\psi_{1}$. Such messages are said pro the message $\psi_{1}$;

- $F_{\text {attack }}: \psi_{1} \rightarrow \forall \psi \in \Psi, \operatorname{tr} \psi=\psi \cap F_{\text {disapproval }}\left(\psi_{1}\right)$, is a function that returns the messages attacking $\psi_{1}$. Such messages are said cons the message $\psi_{1}$.

Let us define a function which returns the messages sent by user $e n_{1}$ : 
- Fentby $: e n_{1} \rightarrow \forall \psi \in \Psi, e n_{\psi}=e n_{1}$, is a function that returns all the messages sent by user $e n_{1}$.

Let us define a function which returns all evaluations done by user $e n_{1}$ :

- Fevaluatedby $: e n_{1} \rightarrow \forall \xi \in \Xi, e n_{\xi}=e n_{1}$, is a function that returns all evaluations done by user $e n_{1}$.

Let us define a function which returns all messages evaluated by user $e n_{1}$ :

- $F_{m s g e v a l u a t e d b y}: e n_{1} \rightarrow \forall \psi \in \Psi, \forall \xi \in F_{\text {evaluatedby }}\left(e n_{1}\right), \psi_{\xi}=\psi$, is a function that returns all messages evaluated by user $e n_{1}$.

Let us define a function which returns the evaluation given to a message $\psi_{1}$ by user $e n_{1}$ :

- Fusermessageevaluation $: e n_{1}, \psi_{1} \rightarrow \forall \xi \in F_{\text {evaluatedby }}\left(e n_{1}\right), \psi_{\xi}=\psi_{1}$, is a function which returns the evaluation given to a message $\psi_{1}$ by user $e n_{1}$.

Let us now define a function to identify leaf messages sent during a human discussion:

- $F_{\text {last }}: \Psi \rightarrow \forall \psi \in \Psi, F_{\text {reinforcement }}(\psi) \cup F_{\text {attack }}(\psi)=\varnothing$, is a function that returns the messages that are not attacked or reinforced thus being leaf messages exchanged in a human discussion.

Let us now define a function that returns all the messages done until message $\psi_{1}$ which can be more than one reinforcement or just only one attack:

- $F_{\text {reinforcementrow }}: \psi_{1} \rightarrow \forall \psi \in \Psi,\left\{\begin{array}{l}F_{\text {reinforcementrow }}(\psi), \psi \in F_{\text {reinforcement }}\left(\psi_{1}\right) \\ \psi_{1}, \psi \in F_{\text {attack }}\left(\psi_{1}\right) \\ \psi_{1}, \psi \notin F_{\text {attack }}\left(\psi_{1}\right) \vee F_{\text {reinforcement }}\left(\psi_{1}\right)\end{array}\right.$,

is a function that returns all the reinforcement or the attack done until $\psi_{1}$.

Let us now define a function that returns all the messages prior to each last message in a human discussion that are either reinforcements (in the limit it could be all reinforcements until the initial message) or an attack:

- Flastdiscussions $: \Psi \rightarrow \forall \psi \in F_{\text {last }}, F_{\text {reinforcementrow }}(\psi)$, is a which returns all the messages prior to each last message that are either reinforcements or an attack.

Let us define a function that returns all the last evaluations done by user $e n_{1}$ :

- Flastevaluatedby $: e n_{1} \rightarrow \forall \xi \in F_{\text {evaluatedby }}\left(e n_{1}\right), \psi_{\xi} \in F_{\text {lastdiscussions }}$, is a function that returns all the last evaluations done by user $e n_{1}$.

In order to identify all the last evaluations done by $e n_{1}$ in a certain dialogue, the $i d_{\psi}$ can be used to filter those evaluations, and therefore the function which returns all the leaf evaluations done by user $e n_{1}$ in a dialogue with $i d_{\psi_{1}}$ would be: 
- $F_{\text {lastevaluationsin }}: i d_{\psi_{1}} \rightarrow \forall \xi \in F_{\text {lastevaluatedby }}\left(e n_{1}\right), i d_{\psi_{\xi}}=i d_{\psi_{1}}$, is a function that returns all the last evaluations done by user $e n_{1}$ for the human dialogue with the id $i d_{\psi_{1}}$.

In (Rahwan et al, 2009) the researchers state that argumentation-based decision process can be decomposed into the following steps: (1) Constructing arguments infavor/against statements (beliefs or decisions), (2) Evaluating the strength of each argument, (3) Determining the different conflicts among arguments, (4) Evaluating the acceptability of arguments and (5) Comparing decisions on the basis of relevant "accepted" arguments. We consider that in our work, we are able to deal with all of this topics as we will prove in our experiments. In addition, our model has the advantage of integrating those points in a perspective that deals with both agents and humans using the same definition.

It will be next described each step for agents to take advantage of the information exchanged during human dialogues.

When an agent is starting a new discussion it will first select the highest evaluation whose representing decision-maker evaluated from all topics that were created. For that, it is next shown a function that returns all root messages from $\Psi$ set:

- Froot $: X \rightarrow \forall \psi \in \Psi, t r_{\psi}=n u l l$, is a function that returns all root messages.

Let us now define a function which returns the root message from the same topic of a message $\psi_{1}$ :

- $F_{\text {topicofmessage }}: \psi_{1} \rightarrow \forall \psi \in \Psi,\left\{\begin{array}{l}F_{\text {topicofmessage }}(\psi), t r_{\psi_{1}}=\psi \wedge \psi \notin F_{\text {root }} \\ \psi, t r_{\psi_{1}}=\psi \wedge \psi \in F_{\text {root }}\end{array}\right.$,

is a function which returns the root message from the same topic of a message $\psi_{1}$.

Let us now define a function which returns all messages from a topic whose root message is $\psi_{1}$ :

- $F_{\text {messagesintopic }}: \psi_{1} \rightarrow \forall \psi \in \Psi, F_{\text {topicofmessage }}(\psi)=\psi_{1}$, is a function which returns all messages from a topic whose root message is $\psi_{1}$.

Let us now define a function which returns all evaluations by a user $e n_{1}$ for a topic whose root message is $\psi_{1}$ :

- $F_{\text {evaluationsintopic }}: \psi_{1}, e n_{1} \rightarrow \forall \xi \in F_{\text {evaluatedby }}\left(e n_{1}\right), \psi_{\xi} \in F_{\text {messagesintopic }}\left(\psi_{1}\right)$, is a function which returns all evaluations by a user $e n_{1}$ for a topic whose root message is $\psi_{1}$.

In order to select the highest evaluation $\xi_{\text {highest }}$ given by an agent $e n_{1}$ we can perform algorithm (1).

After identifying the highest evaluation, the agent should express whether he agrees or disagrees with the corresponding evaluated topic.

Let us define a function which returns a sorted list of messages exchanged prior to a message $\psi_{1}$ until the root message: 
Let en $n_{1}$ be the agent;

Let maxeval be the highest evaluation value;

Let $m$ be a float;

Let $\xi_{\text {highest }}, \xi_{1}$ be two evaluations;

begin

maxeval $\leftarrow 0$;

$\xi_{\text {highest }} \leftarrow N$ ull;

foreach $\psi \in F_{\text {root }}$ do

$\xi_{1} \leftarrow N$ ull

$m \leftarrow 0$

foreach $\xi \in F_{\text {evaluationsintopic }}\left(\psi, e n_{1}\right)$ do

if $e v_{\xi} \geq m$ then

$m \leftarrow e v_{\xi i}$

$\xi_{1} \leftarrow \xi$

end if

end foreach

if $m \geq$ maxeval then

maxeval $\leftarrow m$;

$\xi_{\text {highest }} \leftarrow \xi_{1}$;

end if

end foreach

end

Algorithm 1: Select the highest evaluation of all messages

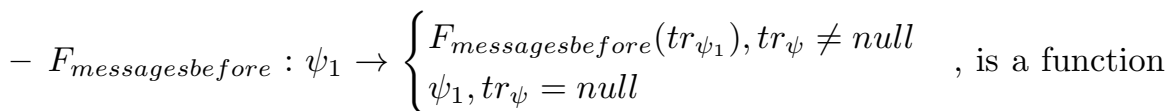

which returns a sorted list of messages exchanged prior to a message $\psi_{1}$ until the root message.

Let us now define a function which returns the direction value of a given message $\psi_{1}$. It returns 1 in case $D r_{\varphi_{\psi_{1}}}=$ infavour and -1 in case $D r_{\varphi_{\psi_{1}}}=$ against:

$-F_{\text {direction }}: \psi_{1} \rightarrow\left\{\begin{array}{l}1, D r_{\varphi_{\psi_{1}}}=\text { infavour } \\ -1, D r_{\varphi_{\psi_{1}}}=\text { against }\end{array} \quad\right.$, is a function which returns the direction value of a given message $\psi_{1}$.

To know if an agent agrees or disagrees with a certain topic, with a root message $\psi_{\text {root }}$, depending on the highest evaluation given to that topic we can perform algorithm (2).

The remaining agents can respond to the message created in the previous algorithm and may extend the discussion on the same branch whether there is more relevant information that can be exchanged or not depending if the message is a leaf in that branch.

Let us define a function which returns the evaluation done by an agent $e n_{1}$ for a message $\psi_{1}$. It returns 1 in case the agent agrees with the message, -1 if the agent disagrees with the message:

- $F_{\text {messageevaluation }}: \psi_{1}, e n_{1} \rightarrow \forall \xi \in F_{\text {evaluatedby }}\left(e n_{1}\right)$, $\left\{\begin{array}{l}1, \psi_{\xi} \wedge \xi \in F_{\text {approval }}\left(\psi_{1}\right) \\ -1, \psi_{\xi} \wedge \xi \in F_{\text {disapproval }}\left(\psi_{1}\right)\end{array} \quad\right.$, is a function which returns the evaluation done by an agent $e n_{1}$ for a message $\psi_{1}$. 


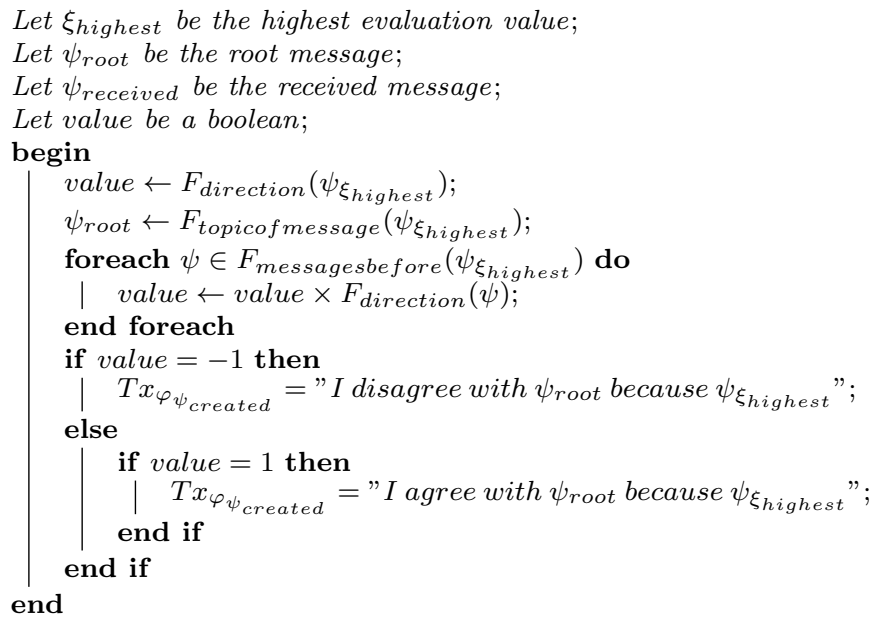

Algorithm 2: Message creation

The algorithm (3) shows how an agent can respond to a message $\psi_{\text {received }}$ in a branch in case that message is one of the leaf messages exchanged.

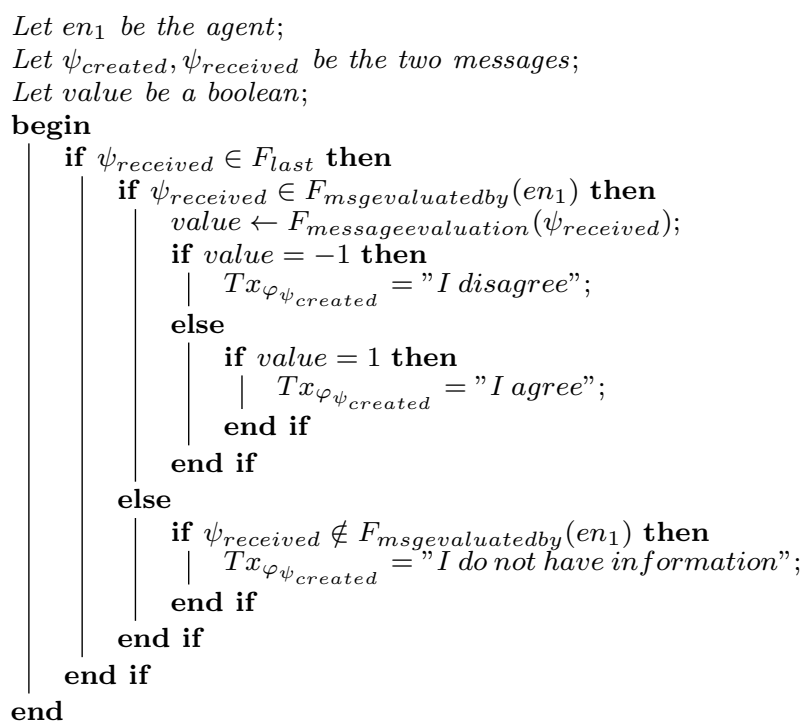

Algorithm 3: Response to leaves' messages

In the case the agent has to respond to a message $\psi_{\text {received }}$ and that message is not one of the leaf messages exchanged, then $\psi_{\text {root }}$ becames $\psi_{\text {received }}$ and the highest evaluation $\xi_{\text {highest }}$ after that message is measured using the algorithm (4). 


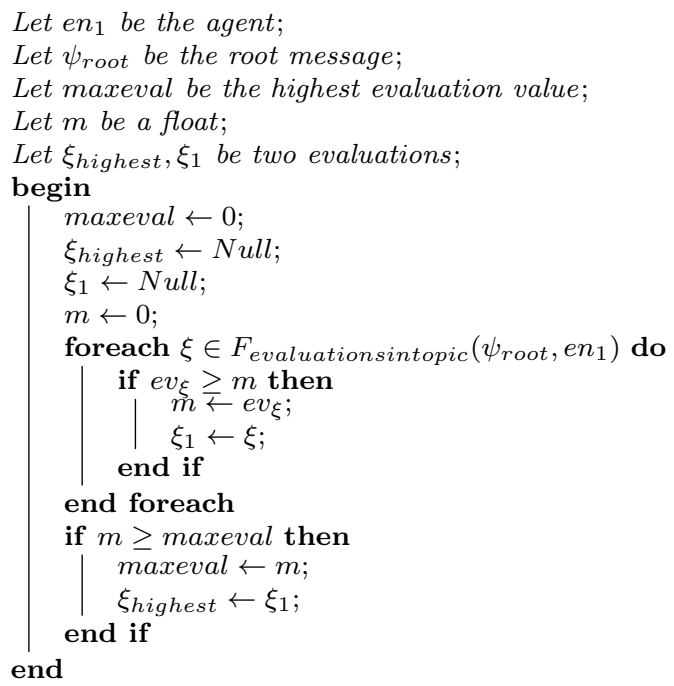

Algorithm 4: Message's children evaluations analysis

After identifying the $\xi_{\text {highest }}$ value, agent $e n_{1}$ responds using the algorithm (5).

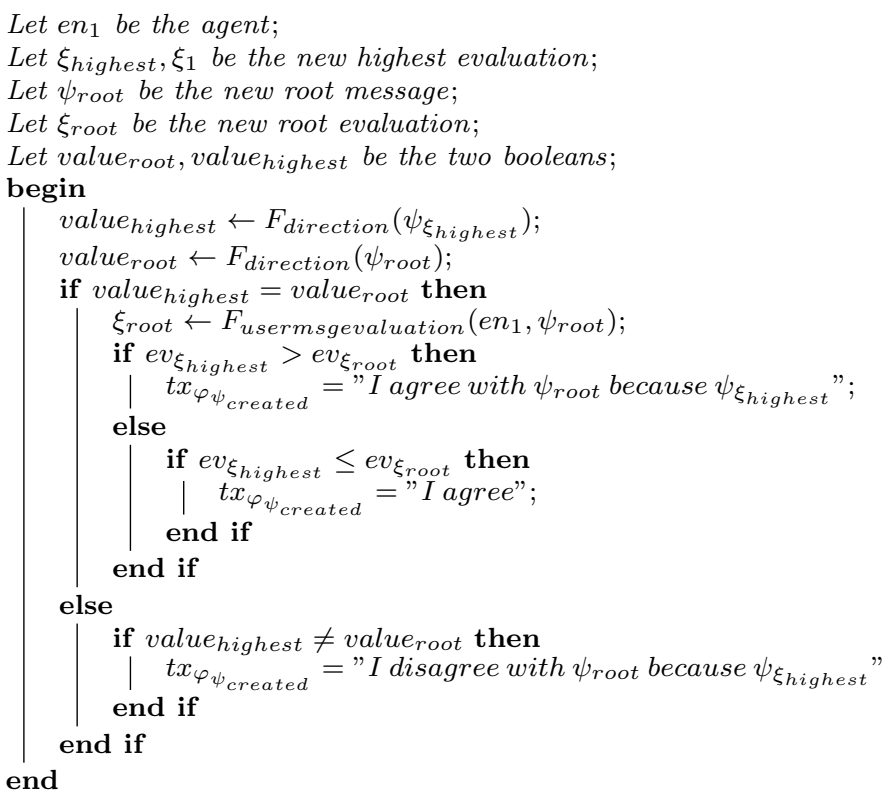

Algorithm 5: Response to non leaves' messages 
The response process repeats until there are only last messages to be discussed and agents must obligatorily respond with either "Agree" or "Disagree" or "With no Information" thus not being able to extend the conversation in that branch. The discussion on the same topic ends if no agent intends to discuss more information existing in different branches of the same topic. Otherwise the agent must repeat algorithm (4) to identify $\xi_{\text {highest }}$ value and then repeat algorithm (2) only if $\psi_{\xi_{\text {highest }}}$ has not been discussed before.

\section{Experiments}

To validate our approach, we have implemented the proposed argumentation model and a multi-agent decision-making dialogue system using JADE (Bellifemine et al, 1999). In addition, we developed a Graphical User Interface (GUI), to the decision-makers interact with the system. The main goal of the performed experiments was to validate each hypothesis defined in this work: (1) make it possible for both agents and decision-makers to use a dialogue which is clear to everyone involved in the decision-making process (2) let both agents and decision-makers to take advantage of the knowledge which is generated and (3) not force decision-makers with information formulation patterns which may compromise the usability of the entire system as well as the process.

We have used a very simple scenario where decision-makers would feel comfortable with the chosen topic. The idea was to put 4 persons together (which represented each decision-maker) with the goal to choose a certain car model that would be purchased by an organization (each decision-maker was told that it would be purchased 300 units of the chosen model). For this work it is not relevant to perform demographic analysis as it is not intended to evaluate the quality of the decision, and these experiments will be only focused on each hypothesis that was introduced.

However, all participants were graduated and aged between 23 and 45 years. Each agent intended to represent one member of the organization administration board. This means that agents are cooperative because they all have to choose the best decision for that organization and they are also competitive because they aim to persuade other agents to accept what they believe that is the best decision (according to their configuration).

Table 2 represents the multi-criteria problem in question. There have been identified 4 possible alternatives.

Table 2: Multi-criteria problem

\begin{tabular}{llllllll}
\hline Alternative & Price & Con & CO2 & CC & HP & TS & Fuel \\
\hline Brand_A1p & 19100 & 3,3 & 87 & 1248 & 95 & 182 & Diesel \\
Brand_B2er & 19065 & 3,2 & 82 & 1461 & 90 & 180 & Diesel \\
Brand_C3fr & 17158 & 4,2 & 112 & 1248 & 75 & 165 & Diesel \\
Brand_D4t & 15400 & 4,3 & 106 & 988 & 90 & 180 & Petrol \\
\hline
\end{tabular}


These alternatives have been classified according to 7 criteria (see Table 3 for all the information regarding the criteria). Considering that we do not only evaluate criteria while trying to solve a multi-criteria problem (Carneiro et al, 2015b,a,c), a decision-maker may prefer a certain alternative for subjective or unknown reasons that are not specified in the problem configuration.

Table 3: Criteria Specification

\begin{tabular}{llll}
\hline Criterion & Greatness & Value & Scale \\
\hline Price (Prc) & Numeric & Minimization & Euros \\
Consumption (Con) & Numeric & Minimization & l/100km \\
CO2 & Numeric & Minimization & $\mathrm{g} / \mathrm{km}$ \\
Displacement (CC) & Numeric & Maximization & $\mathrm{Cm} 3$ \\
Horse Power (HP) & Numeric & Maximization & Horses \\
Top Speed (TS) & Numeric & Maximization & $\mathrm{Km} / \mathrm{h}$ \\
Fuel & Classificatory & Without Value & Petrol/Diesel \\
\hline
\end{tabular}

In order to let decision-makers interact with the system 4 basic functions were created: problem configuration (evaluation of alternatives, criteria and style of behaviour), message creation, message evaluation (and response to that message) and the possibility to view results (from the decision-making process as well as other information). The figures 4, 5, 6 and 7 represent the GUI developed to support these functions.

Decision-makers classify both alternatives and criteria using slide bars with values ranging from 0 to 100 (Fig. 4). These slide bars are based on the Visual Analogue Scale (VAS) as many studies have proven that VAS allows obtaining information more quickly and assertively (Price et al, 1983). It is very easy to configure and can be used in many different electronic devices. Besides this, it also enhances the interaction between the user and the system allowing him to compare directly different alternatives and criteria and make more accurate evaluations.

To create a new message, decision-makers can select the variable that will be discussed and insert an argument in favour or against that variable (Fig. 5 ). Once again, this step is very easy and fast to configure and the components are accessible on all kind of electronic devices.

When evaluating received messages, decision-makers can once again use a slide bar and the system will automatically suggest the user respond that message with an attack or reinforcement depending on the given evaluation (Fig. 6). If the user approved the message he will be suggested to reinforce that message. If the user disapproved the message he will be suggested to attack that message. It must be noted that both evaluations and responses are not mandatory and can be done only if the user intends to do so.

As can be seen in these figures, each step requires very simple and intuitive configuration. To perform all experiments, it was requested to each participant (decision-maker) to imagine and reflect on which alternative he would personally consider as the best and only after that reflection that interaction process 


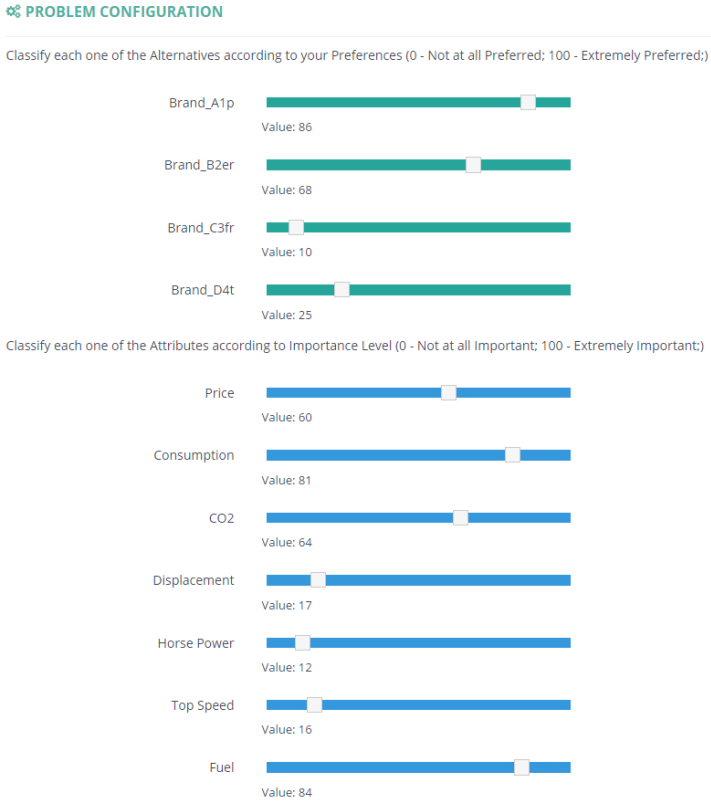

CONFIRM

Fig. 4: Multi-criteria problem configuration's GUI

could be started. It was requested for all participants to configure the problem according to that initial reflection. The results can be seen in Tables 4, 5, 6 and 7 .

Table 4: Participant 1 problem configuration

\begin{tabular}{ll}
\hline Topic & Value \\
\hline Brand_A1p & 0,86 \\
Brand_B2er & 0,80 \\
Brand_C3fr & 0,70 \\
Brand_D4t & 0,40 \\
Price & 0,95 \\
Consumption & 0,99 \\
CO2 & 0,85 \\
Displacement & 0,60 \\
Horse Power & 0,60 \\
Top Speed & 0,40 \\
Fuel & Diesel \\
Agent's behaviour & Integrating \\
Credible Agents & Participant 2, Participant 3 \\
\hline
\end{tabular}




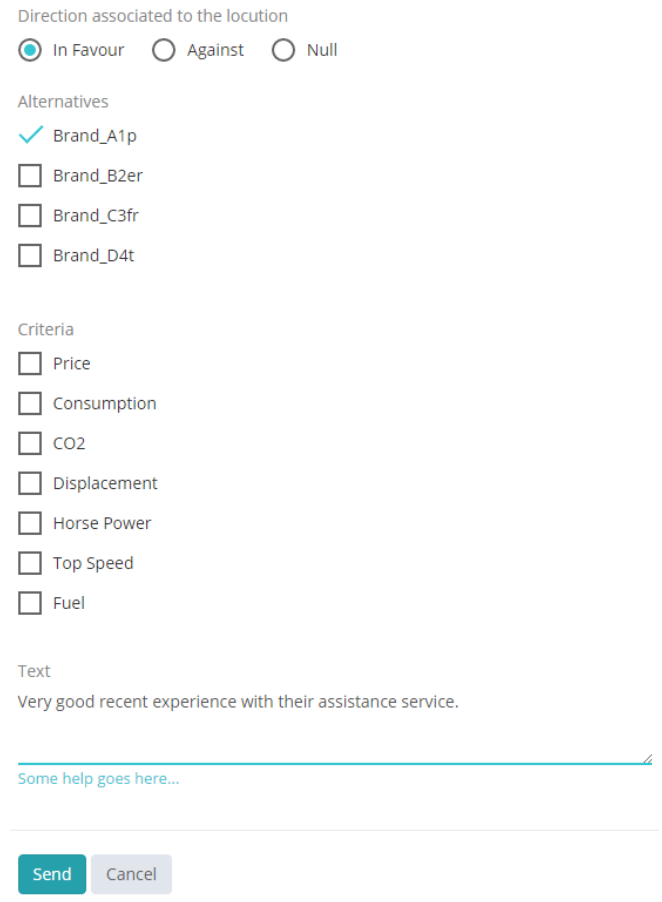

Fig. 5: Message creation GUI

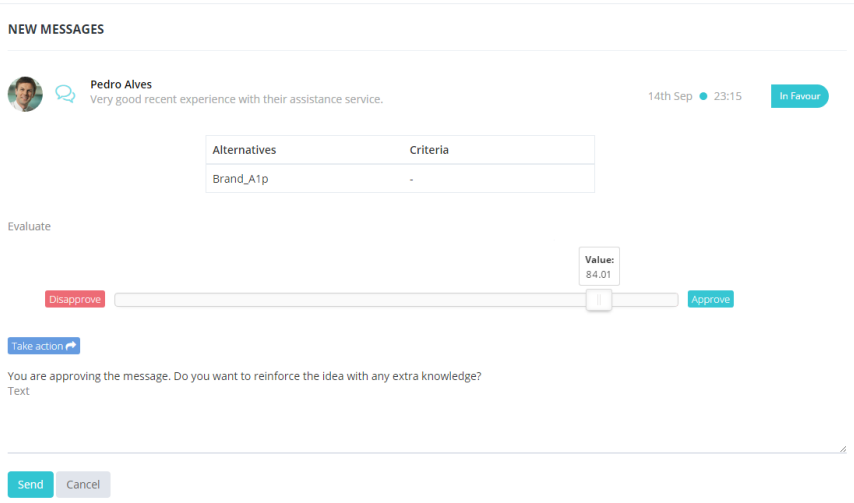

Fig. 6: Received message's evaluation and message's response creation

\subsection{Experiment \#1}

The first experiment studied the way in which agents use the configurations done by decision-makers and the type of information that they can collect and provide to each decision-maker they represent. It is next shown the dialogue 
Table 5: Participant 2 problem configuration

\begin{tabular}{ll}
\hline Topic & Value \\
\hline Brand_A1p & 0,75 \\
Brand_B2er & 0,80 \\
Brand_C3fr & 0,90 \\
Brand_D4t & 0,30 \\
Price & 1,00 \\
Consumption & 0,80 \\
CO2 & 0,50 \\
Displacement & 0,65 \\
Horse Power & 0,65 \\
Top Speed & 0,30 \\
Fuel & Diesel \\
Agent's behaviour & Integrating \\
Credible Agents & - \\
\hline
\end{tabular}

Table 6: Participant 3 problem configuration

\begin{tabular}{ll}
\hline Topic & Value \\
\hline Brand_A1p & 0,57 \\
Brand_B2er & 0,55 \\
Brand_C3fr & 0,20 \\
Brand_D4t & 0,00 \\
Price & 0,90 \\
Consumption & 0,90 \\
CO2 & 0,60 \\
Displacement & 0,80 \\
Horse Power & 0,60 \\
Top Speed & 0,30 \\
Fuel & Diesel \\
Agent's behaviour & Obliging \\
Credible Agents & Participant 2 \\
\hline
\end{tabular}

Table 7: Participant 4 problem configuration

\begin{tabular}{ll}
\hline Topic & Value \\
\hline Brand_A1p & 0,60 \\
Brand_B2er & 0,68 \\
Brand_C3fr & 0,30 \\
Brand_D4t & 0,20 \\
Price & 0,95 \\
Consumption & 1,00 \\
CO2 & 0,56 \\
Displacement & 0,70 \\
Horse Power & 0,45 \\
Top Speed & 0,10 \\
Fuel & Diesel \\
Agent's behaviour & Compromising \\
Credible Agents & - \\
\hline
\end{tabular}




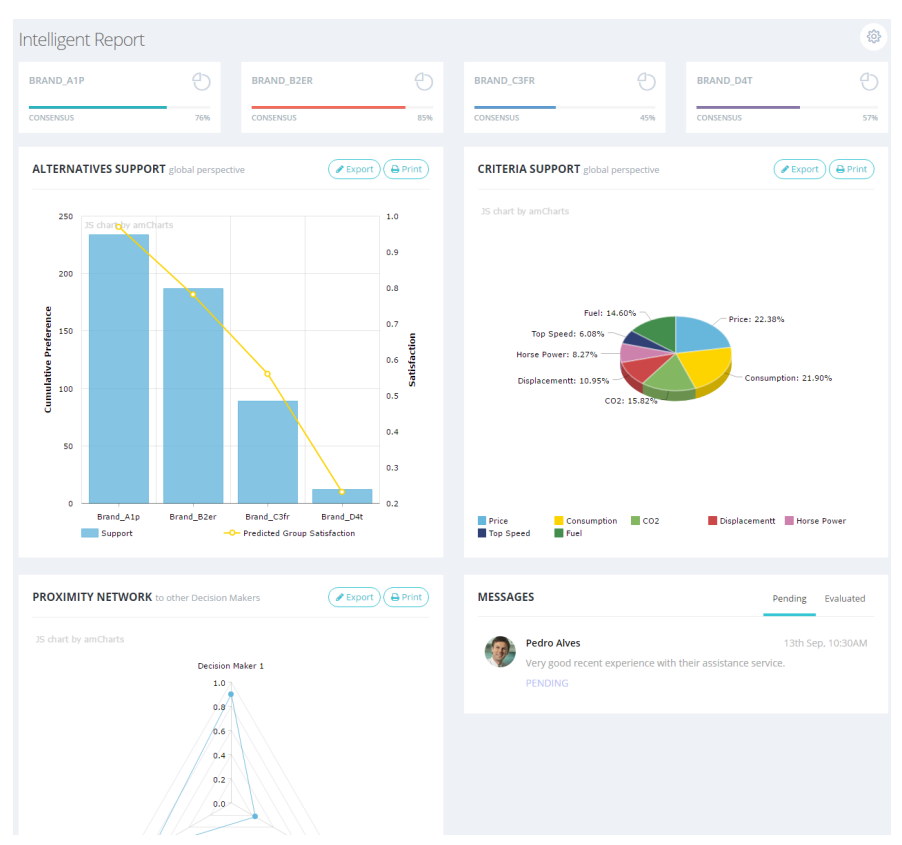

Fig. 7: Results' report

performed between agents, where it is possible to understand the course of the conversation (note: the several types of arguments used in requests as well as the algorithms used to evaluate and accept/decline requests are not the main focus of this work and therefore will not be explored in more detail).

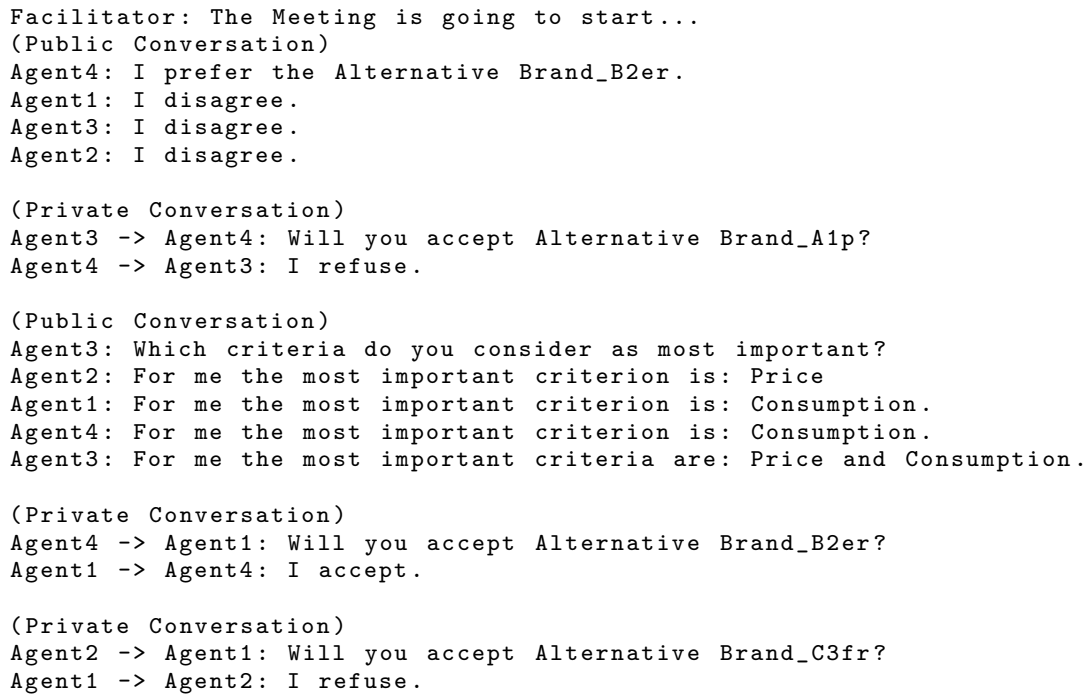




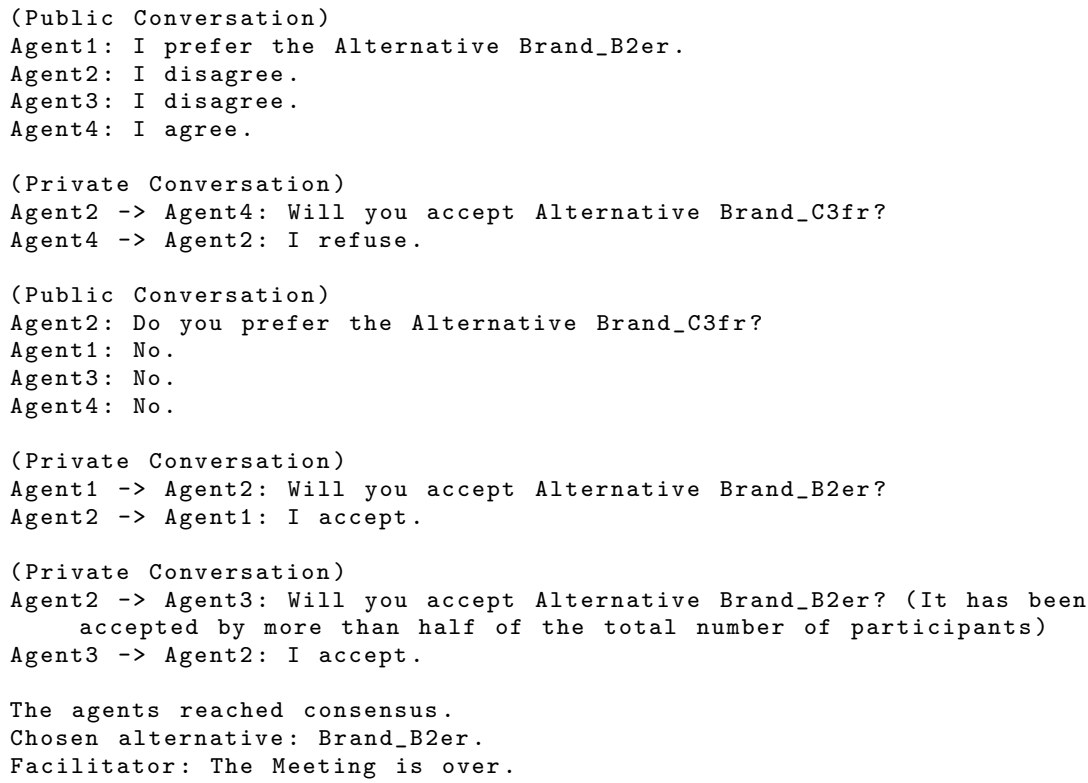

Agents actions were different according to the style of behaviour that was chosen by each representing decision-maker. Agents achieved a consensus towards alternative Brand_B2er. The dialogue is relatively short due to the quickness in which agents were able to achieve the consensus. However, it was still possible for agents to know during each phase of the dialogue the numbers of supporters for each alternative as well as the most preferred criteria. It was also still possible to identify alternatives which were not initially preferred but were still within the acceptation range of participants (always considering the style of behaviour and the goals for the meeting).

\subsection{Experiment \#2}

The second experiment consisted in decision-makers proceeding with the decisionmaking process and use the system to insert new knowledge (creating new messages with arguments) about the problem. In this experiment it was requested for each decision-maker to evaluate all existing messages in order to understand how agents would use the new information shared between decision-makers. The result was the following:

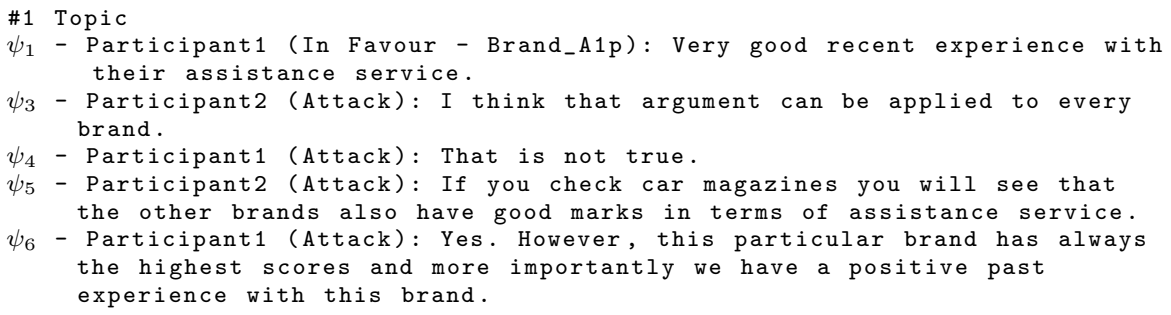


$\psi_{7}$ - Participant4 (Attack): That was verified on previous models. However, if you check the tests regarding the newest models, you will see that the difference is considerable.

$\psi_{2}$ - Participant3 (Reinforcement): Furthermore, I read a study in a car magazine where they considered this brand as the best in terms of assistance service in 2016

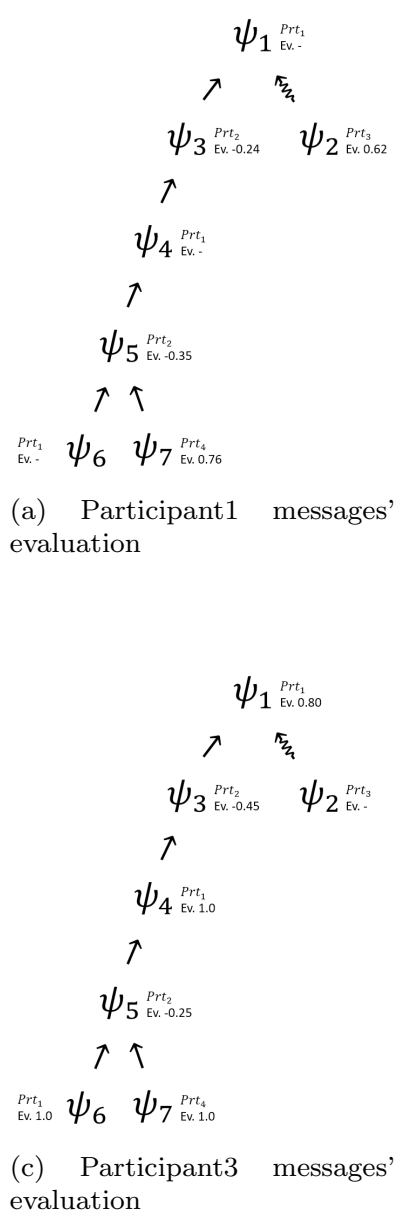

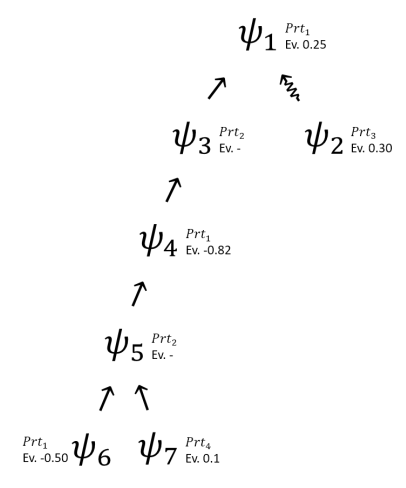

(b) Participant2 messages' evaluation

Fig. 8: Participants' messages evaluation regarding \#1 Topic 


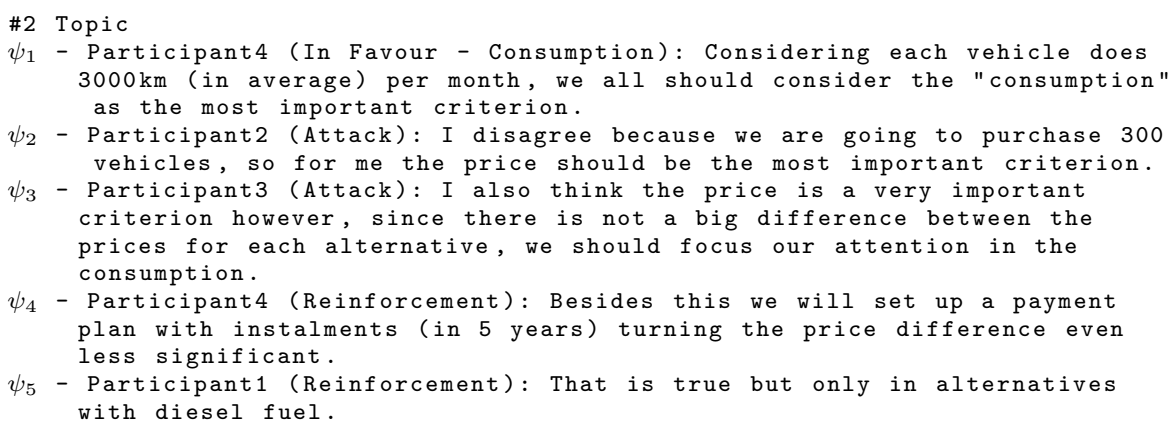

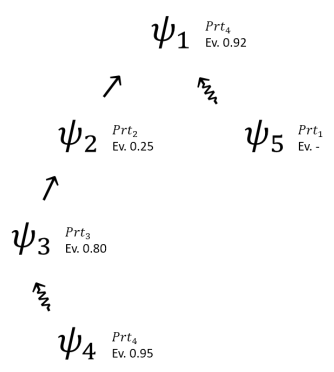

(a) Participant1 messages' evaluation

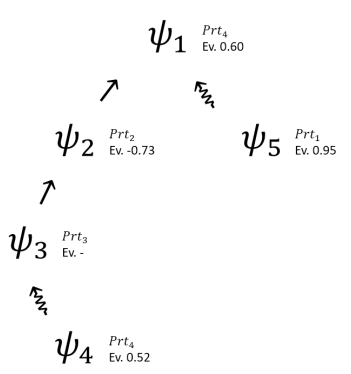

(c) Participant3 messages' evaluation

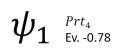

$$
\begin{aligned}
& \psi_{2}^{\substack{P r t_{2} \\
\mathrm{Ek}-\mathrm{F}_{2}}} \psi_{5}^{\substack{P r t_{1} \\
\mathrm{Ev} .0 .43}} \\
& \uparrow \\
& \psi_{3} \begin{array}{c}
\text { Prt } \\
\mathrm{Ev} .0 .15
\end{array} \\
& \text { 会 }
\end{aligned}
$$

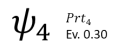

(b) Participant2 messages' evaluation

Fig. 9: Participants' messages evaluation regarding \#2 Topic

After participants (decision-makers) have performed a few conversations (2 topics) the goal was to understand how agents would use the new knowledge in their dialogue. For that a new negotiation round was generated. It is next presented the dialogue performed between agents. It is only shown the part of dialogue which differs from the first experiment. 


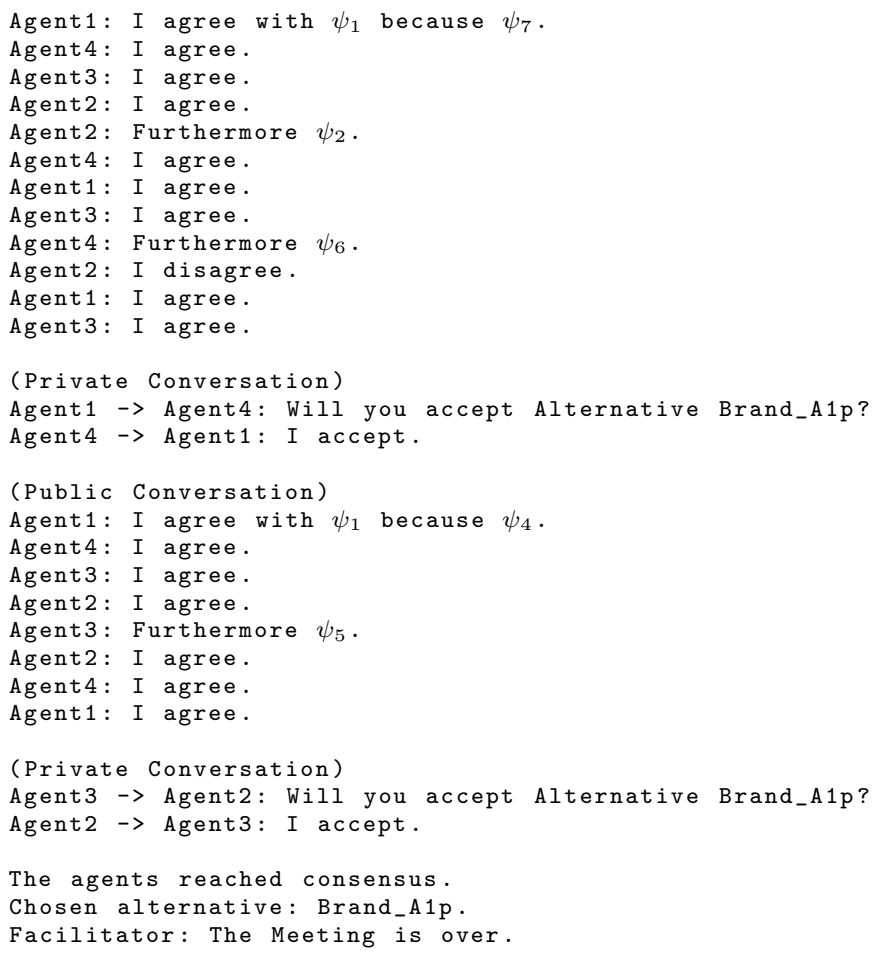

\subsection{Experiment \#3}

In the third experiment it was intended to study how agents would behave in case decision-makers did not evaluate all existing messages. For that it was requested for each decision-maker to only evaluate some existing messages in order to simulate more realistic situations in which decision-makers may not have enough time to completely follow the decision-making process. It should be once again mentioned the main purpose of a group decision support system which is to support the decision process and not replace decision-makers, which means that it is very important that the system is able to provide sustained solutions as the process proceeds further. It should be possible for a decisionmaker which may not have enough time to follow the process closely to still be able to collect and access information at a later time which will allow him to understand how the process evolved and still be able to insert new information as well as reconfigure the problem if it is necessary.

The results obtained in the new evaluations are the following:

Using this more complex scenario with much less available information it was then intended to study how agents would use the new existing knowledge in their dialogue, similarly to the previous experiment. For that, a new negotiation round was generated. It is next show the dialogue performed between 


$$
\begin{aligned}
& \psi_{1}^{\mathrm{Prt}_{\mathrm{fu}}} \\
& \pi \text { ह }
\end{aligned}
$$

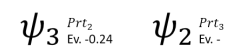

$$
\begin{aligned}
& 7 \\
& \psi_{4}{ }_{4}^{P t_{2}} \\
& \uparrow
\end{aligned}
$$

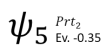

$$
\begin{aligned}
& \uparrow \uparrow
\end{aligned}
$$

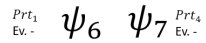

(a) Participant1 messages' evaluation regarding \#1 Topic

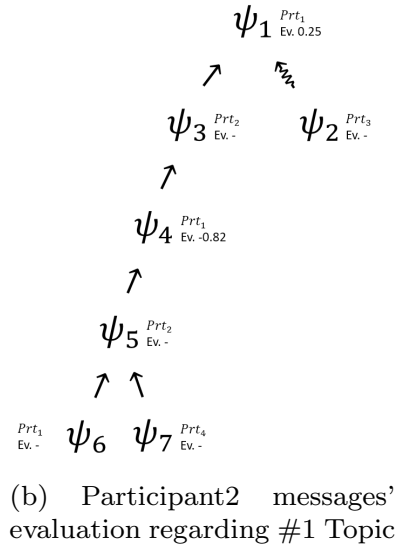

(b) Participant2 messages' evaluation regarding \#1 Topic

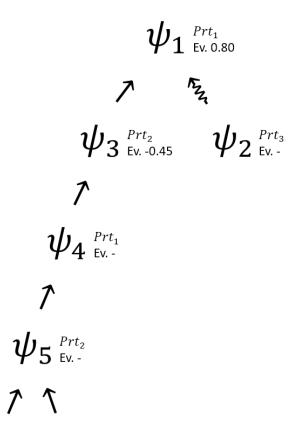

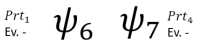

(c) Participant3 messages' evaluation regarding \#1 Topic

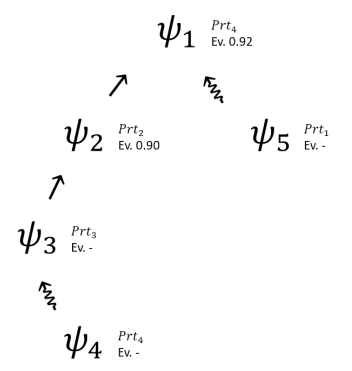

(e) Participant1 messages' evaluation regarding \#2 Topic

$$
\begin{aligned}
& \psi_{1}^{p+p t z} \\
& \text { 万会 }
\end{aligned}
$$

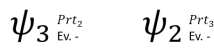

$$
\begin{aligned}
& 7 \\
& \psi_{4} \mathrm{Pr}_{\mathrm{E}} \\
& \uparrow \\
& \psi_{5} \underbrace{\mathrm{Pr}_{2}}_{\mathrm{Ev} .0 .05} \\
& \uparrow \uparrow
\end{aligned}
$$

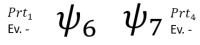

(d) Participant4 messages' evaluation regarding \#1 Topic

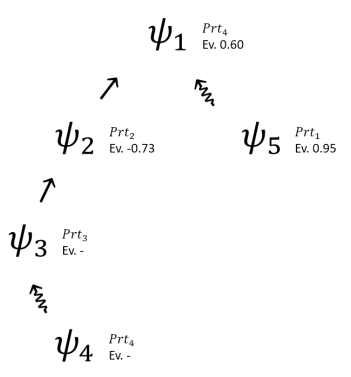

(g) Participant3 messages' evaluation regarding \#2 Topic

$$
\begin{aligned}
& \psi_{1}^{\substack{P r t \\
\text { tru-0.78 }}}
\end{aligned}
$$

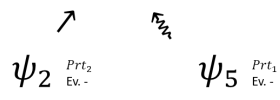

$$
\begin{aligned}
& \uparrow
\end{aligned}
$$

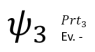

$$
\begin{aligned}
& \text { 会 } \\
& \psi_{4}{ }_{4}^{P r t s}
\end{aligned}
$$

(h) Participant4 messages' evaluation regarding \#2 Topic

Fig. 10: Participants' messages evaluation regarding \#1 Topic and \#2 Topic 
agents. It is only shown the part of the dialogue which differs from the first experiment.

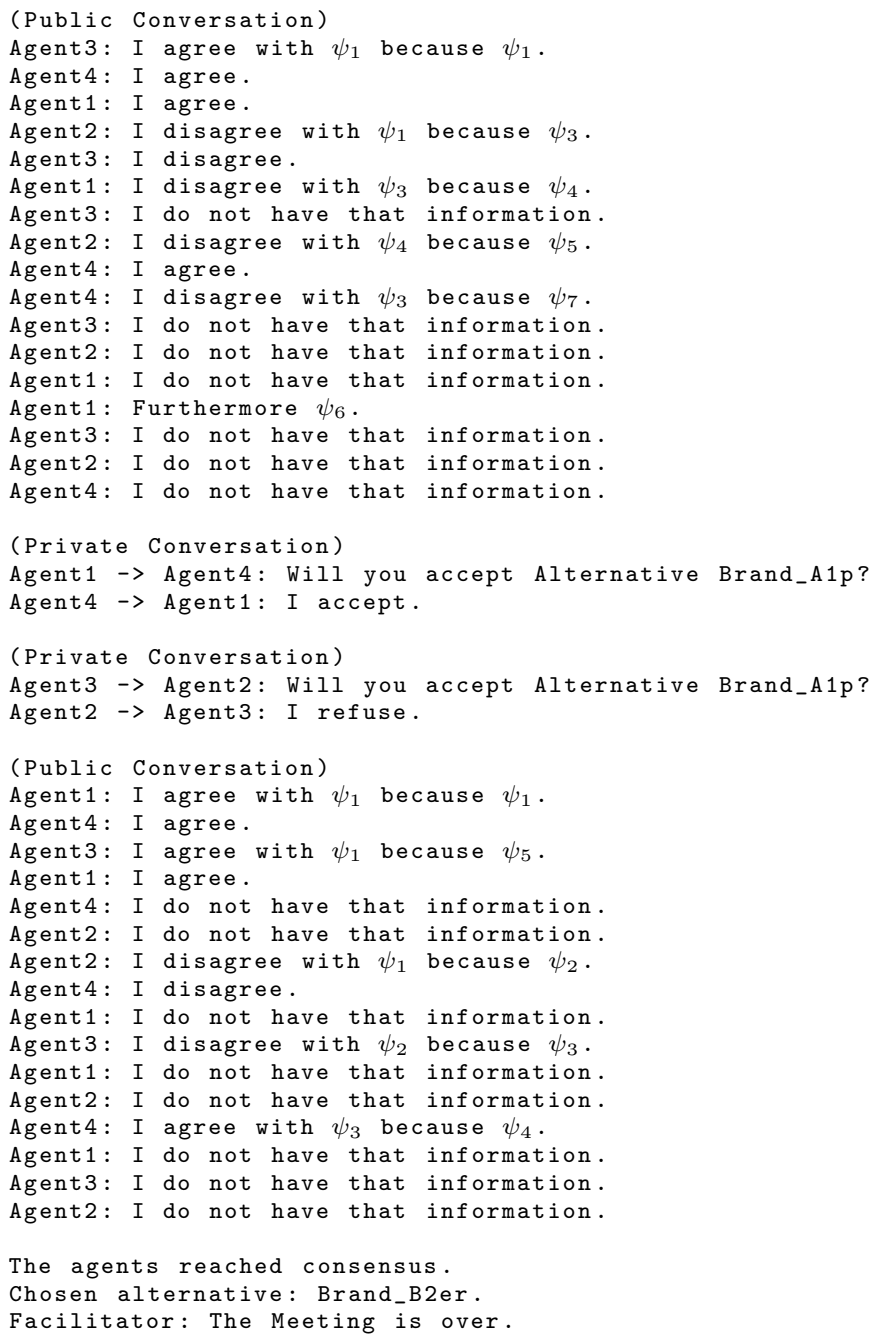

\subsection{Experiment \#4}

In this last experiment random arguments were created in order to build a more complex tree. The idea was to study how agents would use the information existing in a tree with higher complexity compared with the trees that were created in simulations with real participants. The resulting tree as well as its information usage between agents can be seen in figures 11 and 12 .

Tree 1 is the initial tree. Tree 2 corresponds to the selection of most important argument $\left(\psi_{6}\right)$ of the agent $a g_{1}$. When $a g_{1}$ uses $\psi_{6}$ (Tree 3 ) the entire 


$$
\begin{aligned}
& \psi_{1}^{P_{\mathrm{fv}}^{P r_{x}}}
\end{aligned}
$$

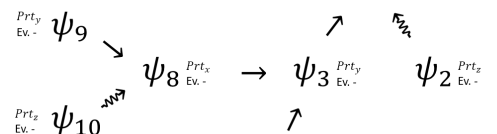

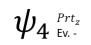

$$
\begin{aligned}
& \uparrow \uparrow
\end{aligned}
$$

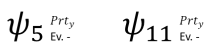

$$
\begin{aligned}
& \uparrow \uparrow \uparrow
\end{aligned}
$$

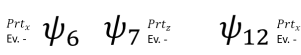

$$
\begin{aligned}
& \text { है } \uparrow
\end{aligned}
$$

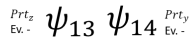

(a) Initial state of the messages' tree

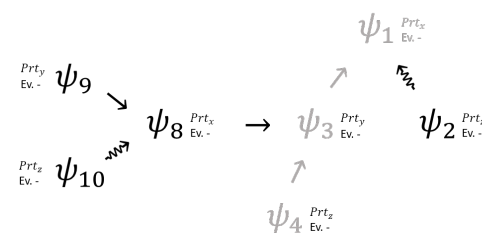

$$
\begin{aligned}
& \uparrow \uparrow
\end{aligned}
$$

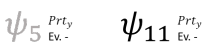

$$
\begin{aligned}
& \uparrow \uparrow \uparrow
\end{aligned}
$$

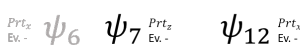

$$
\begin{aligned}
& \text { इ } \uparrow
\end{aligned}
$$

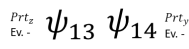

(c) Branch lock

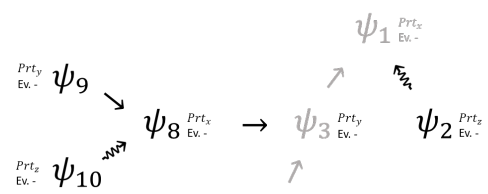

$$
\begin{aligned}
& \psi_{4}{ }_{4}^{p+r_{2}} \\
& \uparrow \uparrow
\end{aligned}
$$

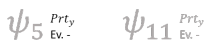

$$
\begin{aligned}
& \uparrow \uparrow \uparrow
\end{aligned}
$$

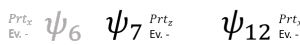

$$
\begin{aligned}
& \text { इ } \uparrow
\end{aligned}
$$

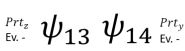

(e) Branch lock

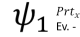

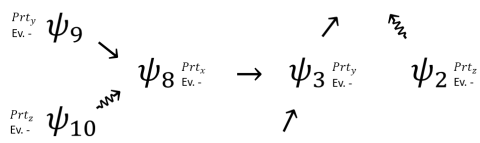

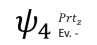

$$
\begin{aligned}
& \uparrow \uparrow
\end{aligned}
$$

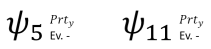

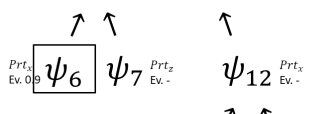

$$
\begin{aligned}
& \text { इ } \uparrow \\
& { }_{\substack{P r_{2} \\
\text { Ev. }}} \psi_{13} \psi_{14} \psi_{14}^{\substack{P r t_{y} \\
\text { Ev. }}}
\end{aligned}
$$

(b) Selection of a message

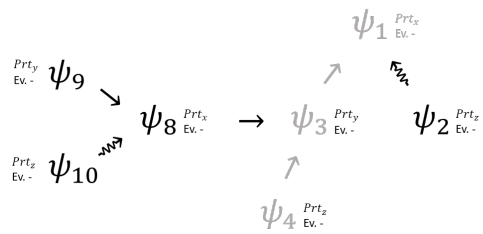

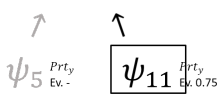

$$
\begin{aligned}
& \uparrow \uparrow \uparrow
\end{aligned}
$$

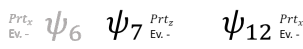

$$
\begin{aligned}
& \text { इ } \uparrow
\end{aligned}
$$

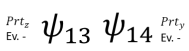

(d) Selection of a message

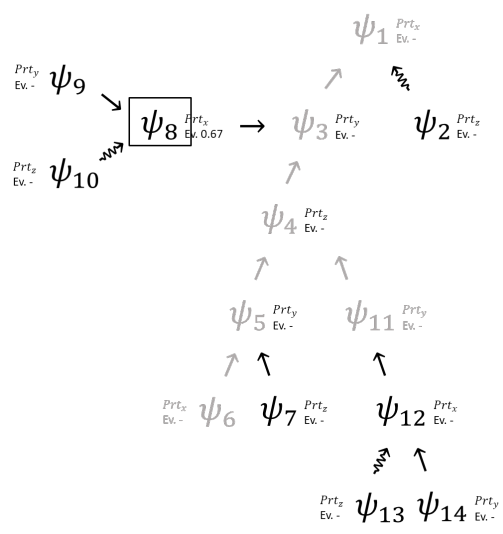

(f) Selection of a message

Fig. 11: Discussion flow - Part 1 


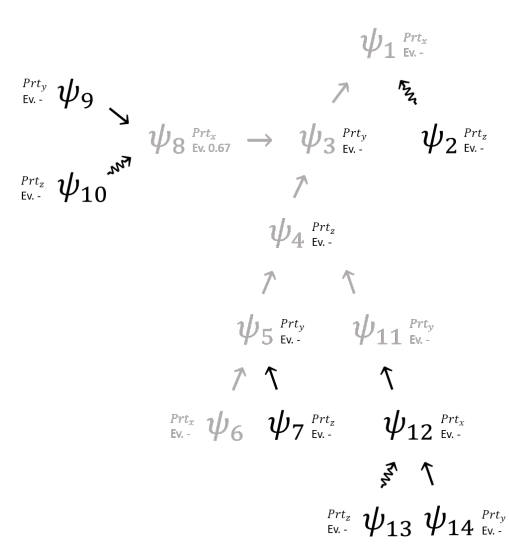

(a) Branch lock

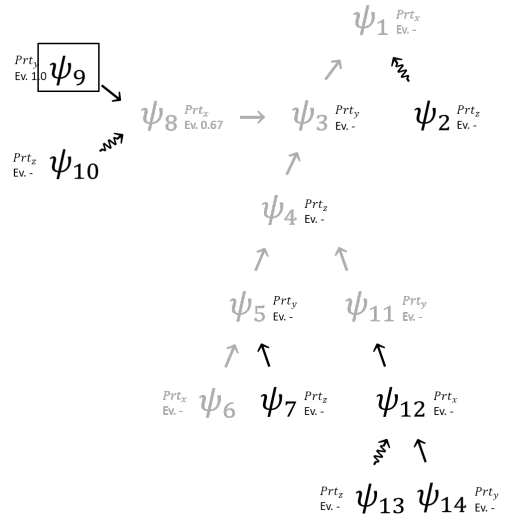

(b) Selection of a message

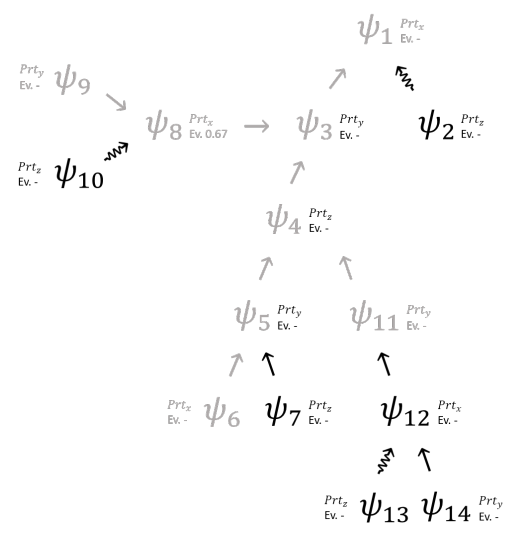

(c) Branch lock

Fig. 12: Discussion flow - Part 2

path from the root message $\left(\psi_{1}\right)$ until $\psi_{6}$ is locked and cannot be used again by other agents. In Tree 4 it is possible to identify an agent $\left(a g_{2}\right)$ which considers the argument in message $\psi_{11}$ as the most important which belongs to a branch that was not discussed before. He will then use that argument (Tree $5)$. Since no other agent considers to be relevant to answer $\psi_{11}$ with an attack or reinforcement, despite $\psi_{11}$ not being the leaf message in that branch, the discussion about it will then end. In Tree 6 we can see that for agent $a g_{4}$ the most important argument in this topic was in message $\psi_{8}$, and therefore, he then uses this argument (Tree 7) to express his opinion on the topic. However, agent $a g_{1}$ disagrees with this argument and performs an attack to message $\psi_{8}$ using message $\psi_{9}$ (Tree 8 and 9). 


\section{Discussion}

In this Section all the hypotheses are discussed. Besides this, some important points are also considered. As mentioned before, the goal of this work is to answer each hypothesis that was previously identified.

\subsection{Experiments Overview}

The first experiment reflects most aspects which are observed in existing argumentation models. It allows creating statistic data and at the same time aim to identify a possible agreement for all agents. We can already consider that our approach differs from this more conventional paradigm since agents are defined with a style of behaviour which allows to better represent the interests of decision-makers. Therefore, we think agents behave in a more dynamic and intelligent way. Nevertheless, this behaviour is not the focus of how work (to learn more please consult (Martinho et al, 2015)).

In the second experiment agents used all information obtained from the first experiment plus the dialogues performed between decision-makers. It was possible to verify that agents used the dialogues performed between decisionmakers successfully. They are able to justify the reasons to support certain preferences as well as identify which agents supported (or not) each argument used by decision-makers. However, since the dialogues used in these experiments were very short, agents used most of the arguments that were not attacked or reinforced. Besides this, since agents could use this dynamic argumentation, they were able to reach a consensus on a different alternative compared to the first experiment.

In the third experiment it was verified that using dynamic argumentation was not very useful as decision-makers evaluated very few messages. The decision achieved was the same of the first experiment. It was still considered as an advantage the fact that new information can be created such as decisionmakers knowing why $a g_{1}$ agrees with message $\psi_{5}$ but at the same time did not evaluate any message attacking it, or which decision-maker does not have an opinion on each message, etc. Finally, in the last experiment, it was necessary to create artificial dialogues with a higher level of complexity in order to learn if agents are able to deal with such kind of context. It was verified that agents are able to "ignore" less relevant messages and create interesting dialogues about only the most relevant messages. The dialogue presented a natural flow which allows agents to take further advantage of this process when making requests in the future.

\subsection{Hypotheses}

The main goal of this work was to validate each hypothesis that was defined: (1) make it possible for both agents and decision-makers to use a dialogue 
which is clear to everyone involved in the decision-making process (2) let both agents and decision-makers to take advantage of the knowledge which is generated and (3) not force decision-makers with information formulation patterns which may compromise the usability of the entire system as well as the process.

This work allowed to validate each hypothesis by observing the results. This means that it was not possible to mathematically validate each hypothesis, however, it seems their validation can still be supported looking at the obtained results for each experiment. It was possible to verify that agents were able (with great success) to use the dialogue performed between decision-makers to create new dialogues and these dialogues offered several advantages to decisionmakers as can be seen in the next section of this work. In terms of configuration costs and looking at the figures presented in previous sections of this work (as well as with the feedback received from the participants in the experiments) they were almost null. When a decision-maker intends to respond to a message he only needs to evaluate that message using a one-click slide bar.

\subsection{Advantages of the proposed approach}

The approach here introduced should be used in a ubiquitous group decision support system. Therefore, in this context, decision-makers do not have much availability or means to gather at the same place and at the same time just to make a decision. It is necessary to use an approach which can suggest intelligent solutions but also report those solutions in a very clear and organized way to each decision-maker saving time and also supporting solutions with explanations that actually let decision-makers learn the reasons behind such suggestions. This allows decision-makers to trust the system and eventually accept the proposed solution or make them look for different options.

Looking at all the reviewed works, it seems that there is not a single proposal that can represent the decision-making process as it happens in face-toface meetings. This is a very important issue that may compromise the success of the system. Furthermore, looking at the negation process itself, some authors refer the need to define a flexible argumentation framework, however, the current proposals cannot suggest a dynamic approach that allows decision-makers to participate in the decision-making process like it happens in face-to-face meetings. The existing approaches only allow to define initial configurations and generate final outputs (undervaluing the process). Besides this, looking at existing proposals, it seems that there is some difficulty to generate information which both decision-makers and agents can understand and use.

The approach presented in this work aims to answer all these necessities. It is the first existing approach in literature completely directed to ubiquitous scenarios which combines both agent and human dialogues. Agents are able to report decision-makers typical questions such as: percentage of agents in favour of each alternative, which agent supports each alternative/ criterion, which criterion is considered as the most important and many other statisti- 
cal data that can be retrieved from initial configurations. Besides this, with the approach here presented agents are able to use the arguments created by decision-makers in their dialogue, and learn (within their necessities) its meaning and importance. It is also possible for agents to report decision-makers the most disagreed points of the discussion which are impeding an eventual consensus. It is possible to know exactly who is in favour of certain alternatives/criteria and also measure, or at least guess, with which level of intensity. This approach allows (due to the type of argumentation that is considered) documenting decisions so that in the future it can be known the reasons that made certain decisions. It is obvious that agents can report the main aspects in favour/against each criterion/alternative and measure the strength/trust for arguments sustaining certain messages, allowing to "ignore" less relevant arguments and building more intelligent reports based on the interests of the decision-makers.

Finally, looking at the definition of the approach, it will be possible to obtain many future advantages which will depend on the work that can be done using the same approach. For example, the intelligence complexity hat can be included in agents. There is enough information that allows agents to perform and accept requests in a much more complex yet interesting way compared to other existing models.

\subsection{Complex or problematic situations}

Some problems may occur under certain situations. One of those is when the decision-maker approves (positively evaluates) an attack done to a message created by him. This situation may seem strange but may still happen. For example, if the message which is attacking contains new knowledge which the decision-maker did not know in first place and he agrees with it. Another problematic situation happens when a decision-maker evaluates (approves or disapproves) two branches with different signs (one branch is attacking the initial message and the other is reinforcing it) with the same value. However, we think that these situations are not too problematic in our approach as the algorithm always considers the message which the decision-maker considered as most important. That message with the highest evaluation is used as reference to know the opinion of the decision-maker towards the root message. We still think that it is fundamental, for this specific situation, that the system should warn the decision-maker in the moment of the evaluation (through the user interface). Furthermore, we even consider that if a decision-maker evaluates a message with a value higher than another message which he had evaluated previously, the system should also warn him asking if he really intends to consider the new message more important than the previous evaluated message. 


\section{Conclusions and Future Work}

Supporting and representing the group decision-making process is a complex work, especially when we consider decision-makers that cannot gather at the same place and time. One of the mechanisms suggested in the literature that supports this type of problem is the automatic negotiation. This mechanism aims to find possible solutions to solve a problem while considering every preference of each decision-maker for that problem.

In this work, we introduced the possibility to create dynamic arguments through a definition of an argumentation model. With our proposal it is possible (and motivates) an interaction between decision-makers, that allows to create new arguments (in favour or against) and also reinforce or attack other arguments created by other decision-makers. The way decision-makers can evaluate each argument will allow the agents to understand the impact of the interactions for the decision-maker they represent and not the content of the conversation (which could be text, sound, etc.). Besides this, since agents share the same problem definition, it allows decision-makers to understand all the interactions between those agents and the reason why they suggest a certain solution for the problem. This approach may also be advantageous to register the reasons that led decision-makers to choose a certain decision. In this work, we also proposed an algorithm that allows agents to navigate and manipulate message trees.

It is always very difficult to validate a proposal of this nature, mainly because we are talking about something that will be used by human beings, and therefore there is not a mathematical proof that grants the acceptance of a model/system. However, our proposal was defined so that it takes advantage of the typical benefits related to group decision-making (unlike the approaches on existent argumentation models that deal with this type of context). We verified that agents were able to use dialogues performed by decision-makers and create/organize new knowledge which in turn is completely perceptible to decision-makers. We verified that our approach takes advantage of the benefits inherent to group decision-making and deals with the decision-making process in a continuous way through several interactions. Besides this, we verified that our approach can be implemented without compromising the system in terms of usability.

As future work, we intend to develop a framework where the information will be analysed using multidimensional matrices. This way agents will be able to use this new knowledge and improve their ability to make requests and consequently evaluate them. Besides this, the agent should also take his defined behaviour into account when doing any analysis in order to properly support and defend the perspective of the decision-maker he represents. Finally, we intend to work on the branch of how and what type of information should be presented to the decision-maker, through what we will call as intelligent reports. 
Acknowledgements This paper is a revised and an expanded version of a paper entitle "Introducing Dynamic Argumentation to UbiGDSS" presented at International Conference on Distributed Computing and Artificial Intelligence 2016, Seville, Spain (Carneiro et al, 2016). This work has been supported by COMPETE Programme (operational programme for competitiveness) within project POCI-01-0145-FEDER-007043, by National Funds through the FCT - Fundação para a Ciência e a Tecnologia (Portuguese Foundation for Science and Technology) within the Projects UID/CEC/00319/2013, UID/EEA/00760/2013, and the João Carneiro PhD grant with the reference SFRH/BD/89697/2012.

\section{References}

Bell DE (1985) Disappointment in decision making under uncertainty. Operations research $33(1): 1-27$

Bellifemine F, Poggi A, Rimassa G (1999) Jade-a fipa-compliant agent framework. In: Proceedings of PAAM, London, vol 99, p 33

Bonzon E, Dimopoulos Y, Moraitis P (2012) Knowing each other in argumentation-based negotiation. In: Proceedings of the 11th International Conference on Autonomous Agents and Multiagent Systems-Volume 3, International Foundation for Autonomous Agents and Multiagent Systems, pp 1413-1414

Booth R, Caminada M, Podlaszewski M, Rahwan I (2012) Quantifying disagreement in argument-based reasoning. In: Proceedings of the 11th International Conference on Autonomous Agents and Multiagent Systems-Volume 1, International Foundation for Autonomous Agents and Multiagent Systems, pp 493-500

Carneiro J, Santos R, Marreiros G, Novais P (2014) Overcoming the lack of human-interaction in ubiquitous group decision support systems. In: Advanced Science and Technology Letters, vol 49, pp 116-124

Carneiro J, Martinho D, Marreiros G, Novais P (2015a) Defining Agents' Behaviour for Negotiation Contexts, Springer, pp 3-14

Carneiro J, Martinho D, Marreiros G, Novais P (2015b) A general template to configure multi-criteria problems in ubiquitous gdss. International Journal of Software Engineering and Its Applications 9:193-206, DOI 10.14257/astl. 205.97.17

Carneiro J, Martinho D, Marreiros G, Novais P (2015c) Individual definition of multi-criteria problems in ubiquitous gdss. Adv Sci Technol Lett

Carneiro J, Santos R, Marreiros G, Novais P (2015d) Ubigdss: A theoretical model to predict decision-makers' satisfaction. International Journal of Multimedia and Ubiquitous Engineering 10:191-200

Carneiro J, Martinho D, Marreiros G, Novais P (2016) Introducing dynamic argumentation to ubigdss. In: Distributed Computing and Artificial Intelligence, 13th International Conference, Springer, pp 471-479

Daume S, Robertson D (2000) An architecture for the deployment of mobile decision support systems. Expert Systems with Applications 19(4):305-318

DeSanctis G, Gallupe B (1985) Group decision support systems: a new frontier. SIGMIS Database 16:3-10, DOI 10.1145/1040688.1040689 
DeSanctis G, Gallupe B (1987) A foundation for the study of group decision support systems. Management Science 33:589-609

Dung PM (1995) On the acceptability of arguments and its fundamental role in nonmonotonic reasoning, logic programming and n-person games. Artificial intelligence $77(2): 321-357$

El-Sisi AB, Mousa HM (2012) Argumentation based negotiation in multiagent system. In: Computer Engineering \& Systems (ICCES), 2012 Seventh International Conference on, IEEE, pp 261-266

Fan X, Toni F (2013) Decision making with assumption-based argumentation. In: International Workshop on Theorie and Applications of Formal Argumentation, Springer, pp 127-142

Fan X, Craven R, Singer R, Toni F, Williams M (2013) Assumption-based argumentation for decision-making with preferences: A medical case study. In: International Workshop on Computational Logic in Multi-Agent Systems, Springer, pp 374-390

Fan X, Toni F, Mocanu A, Williams M (2014) Dialogical two-agent decision making with assumption-based argumentation. In: Proceedings of the 2014 international conference on Autonomous agents and multi-agent systems, International Foundation for Autonomous Agents and Multiagent Systems, pp 533-540

Gordon TF, Karacapilidis N (1997) The zeno argumentation framework. In: Proceedings of the 6 th international conference on Artificial intelligence and law, ACM, pp 10-18

Grudin J (2002) Group dynamics and ubiquitous computing. Communications of the ACM 45:74-78, DOI 10.1145/585597.585618

Hackman JR, Morris CG (1974) Group tasks, group interaction process, and group performance effectiveness: A review and proposed integration. Citeseer

Heras S, Atkinson K, Botti VJ, Grasso F, Julián V, McBurney P (2010) How argumentation can enhance dialogues in social networks. COMMA 216:267274

Heras S, Jordán J, Botti V, Julián V (2013) Argue to agree: A case-based argumentation approach. International Journal of Approximate Reasoning 54(1):82-108

Hill GW (1982) Group versus individual performance: Are $\mathrm{n}+1$ heads better than one? Psychological bulletin 91(3):517

Huber GP (1984) Issues in the design of group decision support systems. MIS Quarterly: Management Information Systems 8:195-204

Ito T, Shintani T (1997) Persuasion among agents: An approach to implementing a group decision support system based on multi-agent negotiation. In: International Joint Conference on Artificial Intelligence, Citeseer, vol 15, pp 592-599

Karacapilidis N, Papadias D (2001) Computer supported argumentation and collaborative decision making: the hermes system. Information systems 26(4):259-277 
Kraus S, Sycara K, Evenchik A (1998) Reaching agreements through argumentation: a logical model and implementation. Artificial Intelligence 104(1):169

Kudenko D, Bauer M, Dengler D (2003) Group decision making through mediated discussions. In: International Conference on User Modeling, Springer, pp 238-247

Kwon O, Yoo K, Suh E (2005) Ubidss: a proactive intelligent decision support system as an expert system deploying ubiquitous computing technologies. Expert Systems with Applications 28:149-161, DOI 10.1016/j.eswa.2004.08. 007

Lamm H, Trommsdorff G (1973) Group versus individual performance on tasks requiring ideational proficiency (brainstorming): A review. European journal of social psychology $3(4): 361-388$

Marey O, Bentahar J, Asl EK, Mbarki M, Dssouli R (2014a) Agents' uncertainty in argumentation-based negotiation: classification and implementation. Procedia Computer Science 32:61-68

Marey O, Bentahar J, Dssouli R, Mbarki M (2014b) Measuring and analyzing agents' uncertainty in argumentation-based negotiation dialogue games. Expert Systems with Applications 41(2):306-320

Marey O, Bentahar J, Khosrowshahi-Asl E, Sultan K, Dssouli R (2015) Decision making under subjective uncertainty in argumentation-based agent negotiation. Journal of Ambient Intelligence and Humanized Computing $6(3): 307-323$

Marreiros G, Santos R, Ramos C, Neves J (2010) Context-aware emotionbased model for group decision making. Intelligent Systems, IEEE 25:31-39, DOI 10.1109/MIS.2010.46

Martinho D, Carneiro J, Marreiros G, Novais P (2015) Dealing with agents' behaviour in the decision-making process. In: Workshop Proceedings of the 11th International Conference on Intelligent Environments, IOS Press, vol 19, p 4

Müller J, Hunter A (2012) An argumentation-based approach for decision making. In: 2012 IEEE 24th International Conference on Tools with Artificial Intelligence, IEEE, vol 1, pp 564-571

Osborn AF (1963) Applied Imagination; Principles and Procedures of Creative Problem-solving: Principles and Procedures of Creative Problem-solving. Scribner

Parsons S, Sklar E, Singh MP, Levitt KN, Rowe J (2013) An argumentationbased approach to handling trust in distributed decision making. In: AAAI Spring Symposium: Trust and Autonomous Systems

Price DD, McGrath PA, Rafii A, Buckingham B (1983) The validation of visual analogue scales as ratio scale measures for chronic and experimental pain. Pain 17(1):45-56

Rahwan I, Simari GR, van Benthem J (2009) Argumentation in artificial intelligence, vol 47. Springer

Sánchez-Anguix V, Botti V, Julián V, García-Fornes A (2011) Analyzing intrateam strategies for agent-based negotiation teams. The 10th International 
Conference on Autonomous Agents and Multiagent Systems 53:929-936

Shaw ME (1932) A comparison of individuals and small groups in the rational solution of complex problems. The American Journal of Psychology 44(3):491-504

Sierra C, Jennings NR, Noriega P, Parsons S (1997) A framework for argumentation-based negotiation. In: International Workshop on Agent Theories, Architectures, and Languages, Springer, pp 177-192

Sklar EI, Parsons S, Li Z, Salvit J, Perumal S, Wall H, Mangels J (2016) Evaluation of a trust-modulated argumentation-based interactive decisionmaking tool. Autonomous Agents and Multi-Agent Systems 30(1):136-173

Watson WE, Michaelsen LK, Sharp W (1991) Member competence, group interaction, and group decision making: a longitudinal study. Journal of Applied Psychology 76(6):803

Van der Weide TL, Dignum F, Meyer JJC, Prakken H, Vreeswijk G (2011) Multi-criteria argument selection in persuasion dialogues. In: International Workshop on Argumentation in Multi-Agent Systems, Springer, pp 136-153

Wyner A, Atkinson K, Bench-Capon T (2012) A functional perspective on argumentation schemes. In: Proceedings of the 9th International Workshop on Argumentation in Multi-Agent Systems (ArgMAS 2012), pp 203-222

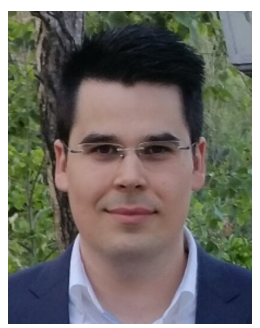

João Carneiro is a PhD student at MAP-i (Minho, Aveiro and Porto Doctoral Program in Computer Science), guest professor in School of Technology and Management and researcher at the Research Group on Intelligent Engineering and Computing for Advanced Innovation and Development (GECAD) and at the ALGORITMI Centre. His main areas of interest are Decision Satisfaction, Multi-Agent Systems, Emotional Agents, Persuasive Argumentation and Group Decision Support Systems. He received his master in informatics from Polytechnic of Porto's Institute of Engineering (ISEP/IPP). During his bacheral and master he received several awards regarding to the work developed. 


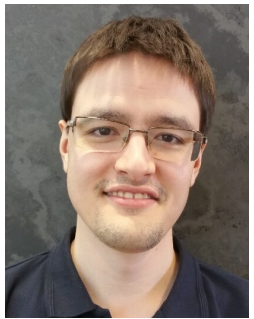

Diogo Martinho is a researcher at the Research Group on Intelligent Engineering and Computing for Advanced Innovation and Development (GECAD). He received his master in informatics from Polytechnic Institute of Porto. His main areas of interest are: Artificial Intelligence, Multi-Agent Systems, Group Decision Support Systems, Argumentation-Based Negotiation and Ubiquitous Computing. His major skills are logic reasoning, language programming and software engineering.

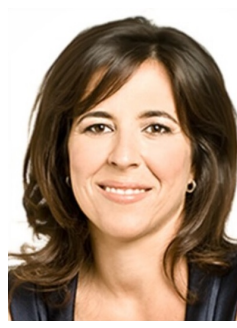

Goreti Marreiros is graduated in Computer Science since 1995, have a master in Information Management (2002) both from the Porto University, and a PhD in Informatics-Artificial Intelligence from Minho University (2008). Since 1997 she is a professor in the Department of Computer Engineering at the School of Engineering of the Polytechnic Institute of Porto (ISEP). Goreti Marreiros is also researcher and sub-director of GECAD - Research Group on Intelligent Engineering and Computing for Advanced Innovation and Development, having participated in over 25 research projects, and actually she is coordinating 4 research projects. In the last years, she has taught and been responsible for a number of courses on Decision Support Systems, Multiagent Systems and on Artificial Intelligence at the graduate and postgraduate level. 


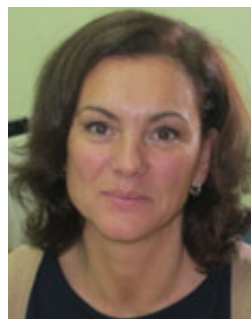

Amparo Jiménez Vivas is Full Professor in Educative and Professional Diagnosis at the Pontifical University of Salamanca, Spain. She holds a PhD in Education and Psychology and her research interests are focused on the diagnosis of educational needs in different environments. She was head of the General Foundation of the Pontifical University of Salamanca from 2012 to 2015. She has published several papers in recognized conferences and Journals.

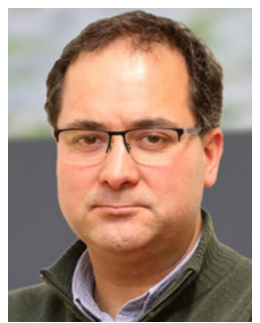

Paulo Novais is an Associate Professor with Habilitation of Computer Science at the Department of Informatics, in the School of Engineering of the University of Minho (Portugal) and a researcher at the ALGORITMI Centre in which he is the coordinator of the research group ISLab - Synthetic Intelligence. He is the director of the PhD Program in Informatics and co-founder and Deputy Director of the Master in Law and Informatics at the University of Minho. His main research aim is to make systems a little more smart, intelligent and also reliable. He is the co-author of over 230 book chapters, journal papers, conference and workshop papers and books. 